\title{
COMPUTATION OF HETEROCLINIC ARCS WITH APPLICATION TO THE VOLUME PRESERVING HÉNON FAMILY
}

\author{
J.D. MIRELES JAMES, HECTOR LOMELÍ
}

\begin{abstract}
Let $f: \mathbb{R}^{3} \rightarrow \mathbb{R}^{3}$ be a diffeomorphism with $p_{0}, p_{1} \in \mathbb{R}^{3}$ distinct hyperbolic fixed points. Assume that $W^{u}\left(p_{0}\right)$ and $W^{s}\left(p_{1}\right)$ are two dimensional manifolds which intersect transversally at a point $q$. Then the intersection is locally a one-dimensional smooth arc $\tilde{\gamma}$ through $q$, and points on $\tilde{\gamma}$ are orbits heteroclinic from $p_{0}$ to $p_{1}$.

We describe and implement a numerical scheme for computing the jets of $\tilde{\gamma}$ to arbitrary order. We begin by computing high order polynomial approximations of some functions $P_{u}, P_{s}: \mathbb{R}^{2} \rightarrow$ $\mathbb{R}^{3}$, and domain disks $D_{u}, D_{s} \subset \mathbb{R}^{2}$, such that $W_{l o c}^{u}\left(p_{0}\right)=P_{u}\left(D_{u}\right)$ and $W_{l o c}^{s}\left(p_{1}\right)=P_{s}\left(D_{s}\right)$ with $W_{l o c}^{u}\left(p_{0}\right) \cap W_{l o c}^{s}\left(p_{1}\right) \neq \emptyset$. Then the intersection arc $\tilde{\gamma}$ solves a functional equation involving $P_{s}$ and $P_{u}$. We develop an iterative numerical scheme for solving the functional equation, resulting in a high order Taylor expansion of the arc $\tilde{\gamma}$. We present numerical example computations for the volume preserving Hénon family, and compute some global invariant branched manifolds.
\end{abstract}

Key words. heteroclinic connections, stable manifold, unstable manifold, volume preserving Hénon Map, Newton Method

AMS subject classifications. 34K19, 34K28, 34K32, 37C05, 37C29, 65P99, 65Q20, 65Q30, 90C53, 15A09

\section{Introduction.}

1.1. Overview. In this work we a develop method for computing heteroclinic connecting orbits between a pair of distinct hyperbolic fixed points of a smooth three dimensional discrete dynamical system. Heteroclinic orbits arise as intersections of the stable and unstable manifolds of these fixed points, and in the present work we are particularly interested in the case where the stable and unstable manifolds are two dimensional and intersect transversally. Near a transverse intersection point, standard dimension counting arguments show that the intersection is locally a one-dimensional smooth manifold, which we call a heteroclinic arc. Given a single point $q$ in the transverse intersection of the stable and unstable manifolds, we develop a method for computing the Taylor expansion of the heteroclinic arc through $q$ to arbitrary order.

Our method depends on the so called Parameterization Method for computing stable and unstable manifolds of fixed points, as presented in $[12,11]$. The first step of our scheme requires explicit numerical implementation of the two dimensional parameterization method for three dimensional maps. Such an implementation has not appeared explicitly in the literature, and is of independent interest. Therefore we describe our implementation in some detail.

Next we show that heteroclinic arcs solve certain functional equations, which depend in turn on the parameterization functions of the stable and unstable manifolds. The heteroclinic functional equations are solved using a Newton iteration scheme on spaces of formal power series, the result of which is a Taylor expansion of the heteroclinic arc. We give explicit recursion relations for the Newton Scheme. 
A schematic for our computational scheme is as follows:

Algorithm 1 (Computation of Heteroclinic Arc).

Step 1: Compute Parameterizations $P_{u}: D_{u} \subset \mathbb{R}^{2} \rightarrow \mathbb{R}^{3}$ and $P_{s}: D_{s} \subset \mathbb{R}^{2} \rightarrow \mathbb{R}^{3}$

having $P_{s}\left(D_{s}\right)=W_{l o c}^{s}\left(p_{1}\right)$ and $P_{u}\left(D_{u}\right)=W_{l o c}^{u}\left(p_{0}\right)$.

Step 2: Compute a single intersection point $q \in W_{l o c}^{u}\left(p_{0}\right) \cap W_{l o c}^{s}\left(p_{1}\right)$.

Step 3: Compute the Taylor expansion of the heteroclinic arc $\tilde{\gamma}$ through $q$.

While our computational scheme in not limited to polynomial or volume preserving maps, it is useful to fix a specific example system. In order to demonstrate the utility of the numerics developed throughout the paper, we consider the volume preserving Hénon family. This is the collection of maps defined by

$$
f(x, y, z)=\left(\begin{array}{c}
f_{1}(x, y, z) \\
f_{2}(x, y, z) \\
f_{3}(x, y, z)
\end{array}\right)=\left(\begin{array}{c}
\alpha+\tau x+z+a x^{2}+b x y+c y^{2} \\
x \\
y
\end{array}\right),
$$

where $a+b+c=1$ (to grantee volume preserving).

The volume preserving Hénon family was introduced in [27]. A thorough discussion of the dynamics of the map, along with a more complete discussion of references can be found in [17]. The relevance of the volume preserving property is to guarantee that a generic fixed point has the kind of stability we are interested in: namely a two dimensional unstable manifold and one dimensional stable manifold or vice versa. The map is quadratic with quadratic inverse.

The paper is organized as follows. The remainder of the introduction reviews the existing literature concerning numerical computation of heteroclinic orbits. Section 2 is devoted to background material and notation used throughout the paper. The main results of the paper are explicit recursion relations for steps one and three of Algorithm 1. These are given in Section 3 and Section 4. Step two of Algorithm 1 is standard finite dimensional computation and is postponed until Section 6. In Section 5 we discuss the determination of appropriate domains for the parameterizations $P_{u}$ and $P_{s}$, and the arc $\tilde{\gamma}$. Section 7 presents example computations for the volume preserving Hénon map. The appendix collects some results about power series of two variables which we exploit throughout the paper.

1.2. Existing Literature and Context of the Current Work. It often happens that one invariant object of a dynamical system induces the existence of another invariant object. For example, the stable manifold theorem [37], implies that if one has a normally hyperbolic invariant manifold, there are two associated invariant manifolds: the stable and unstable manifolds. These provide important information about the global dynamics of a system: the existence of a transverse intersection of two of these stable and unstable manifolds is associated with the onset of chaos, and in many situations gives rise to the famous horseshoe construction of Smale. These intersections are called homoclinic or heteroclinic, depending on wether they appear between one object or several.

Frequently, in order to study the structure of a model, one can study the existence of homoclinic and heteroclinic connections between equilibria (as in Morse Theory $[34,44,43])$, or in general, between normally hyperbolic invariant manifolds. 
One common situation is that these intersections appear after a perturbation of an integrable completely degenerate intersection called saddle connection. The PoincaréMelnikov method $[20,33,38]$ is a widely used technique for detecting such intersections. Unfortunately, the method is limited to the very special situation that was described above: it only detects some specific transversal intersections that exist close to a degenerate system. Many interesting situations are not of this type.

In the past, a great number of authors have studied the problem of finding reliable numerical approximations of heteroclinic dynamics. Since heteroclinic dynamics arises naturally at the intersection of stable and unstable manifolds, the question of numerical approximation of the stable and unstable manifolds is closely related to the question of numerically approximating heteroclinic dynamics. While an exhaustive review of the literature is beyond the scope of the present work, we mention a number of developments most closely related to the present work.

Several methods have been developed to compute one-dimensional invariant manifolds for two- dimensional dynamical systems (both maps and ODEs). One of the first works that considered efficient numerical methods to compute invariant manifolds of two dimensions was [24]. It is just recently that some authors [15, 22, 26, 18] have studied the higher dimensional problem and, in particular, the efficient computation of homoclinic and heteroclinic intersections. If one considers, for instance, a three-dimensional autonomous ODE, a possible heteroclinic connection could consist of a one-dimensional manifold: the orbit of a single point. Then the main difficulty in this case is to find an initial condition for such an orbit.

In [15], the authors propose some methods for the numerical continuation of point-to-cycle heteroclinic connecting orbits in a three-dimensional system of ODEs. They recognize that finding heteroclinic orbits by continuation of connecting orbits in ODE systems has been notoriously difficult. Previous work on the case of heteroclinic connecting orbits between hyperbolic points include [16]

In [46], the existence and properties of discrete embedded solitons is studied. The problem appears to be equivalent to that of the existence of a homoclinic orbit in a four-dimensional diffeomorphism. In [13] shadowing techniques are used to prove the existence transversal homoclinic and heteroclinic orbits in higher dimensional maps. This shadowing technique includes a suitable numerical approximation.

A more complicated problem is to find heteroclinic connections for diffeomorphisms in dimension three and higher. The added dimension allows for the possibility of non-isolated heteroclinic orbits. In this paper, we present a method to find onedimensional heteroclinic intersections, or heteroclinic arcs, for a family of quadratic diffeomorphisms. Non-isolated heteroclinic orbits can arise as the transverse intersection of two dimensional invariant manifolds, and have been discussed from a theoretical point of view for example in [14, 1, 2, 29, 31, 3, 9].

Previous numerical work in this direction includes [8], in which the authors study higher dimensional maps and propose a method in which they approximate infinite homoclinic orbits by an orbit segments of finite length that satisfy a finite boundary value problem. Other possibilities are studied in [4, 21, 47]. Many of the previous methods are based on manipulation of grid approximations of these manifolds. An important survey of these methods appeared in [25]. 
Previous work that uses Taylor approximations for invariant manifolds appeared in [39]. Some recent work which uses high order Taylor methods to compute stable and unstable manifolds of planar diffeomorphisms is [35]. In fact, our methods lie very much in the automatic differentiation paradigm (see for example $[32,7,6]$ or $[10]$ for a much more thorough review of the automatic differentiation literature). A general purpose software package for carrying out automatic differentiation computations with rigorous enclosure of remainders is the COSY package [5]. For fuller discussion of practical algorithms for manipulating polynomials, a standard reference is [23]. Finally we mention also the related work of $[36,41,42]$ on numerical approximation of manifolds defined implicitly as the solution of algebraic equations, and the software package MANPAK [40] for computing such objects.

\section{Preliminaries and Notation.}

2.1. Linear Algebra. The following can be found in any text on applications of linear algebra. See for example [45].

Definition 2.1. Let $A$ be an $m \times n$ matrix of real numbers. A singular value decomposition of $A$ is a triple of matrices $(U, \Sigma, V)$ having the following properties.

a) $A=U \Sigma V^{T}$.

b) $U$ is an $m \times m$ orthogonal matrix.

c) $V$ is an $n \times n$ orthogonal matrix.

d) $\Sigma$ is an $m \times n$ diagonal matrix with $s_{i i} \geq 0$, and $s_{i j}=0$ if $i \neq j$.

e) There is a $0 \leq K \leq m$ so that $s_{i i}>0$ when $1 \leq i \leq K$. If $K>0$ nonzero values of $\Sigma$ are ordered $s_{11} \geq \ldots \geq s_{K K}>0$.

f) The last $n-K$ columns of $V$ form an orthonormal basis for the $\operatorname{ker}(A)$. The first $K$ vectors of $V$ form an orthonormal basis for $\operatorname{ker}(A)^{\perp}$

g) The first $K$ columns of $U$ for an orthonormal basis for range $(A)$. The last $m-K$ columns of $U$ are a basis for range $(A)^{\perp}$.

h) $\|A\|=s_{11}$.

For further discussion and proofs of the claims, see [45]. For a given real matrix $A$ we use the notation

$$
(U, \Sigma, V)=\operatorname{SVD}(A)
$$

to denote the function which returns a singular value decomposition for $A$. The singular value decomposition of an $m \times n$ matrix always exists [45]. We make use of the algorithms embedded in MatLab, which are based on the LINPAC implementations.

Definition 2.2. Let $A$ be an $m \times n$ real matrix and $(U, \Sigma, V)=\operatorname{SVD}(A)$. The Moore-Penrose pseudoinverse of $A$, which we denote by $[A]_{M P}^{-1}$, is the real $n \times m$ matrix defined by

$$
[A]_{M P}^{-1}=V \Sigma^{+} U^{*}
$$

where $*$ denotes the conjugate transpose and $\Sigma^{+}$is obtained by taking the reciprocals of the non-zero diagonal elements of $\Sigma$.

Given and equation of the form $A x=b$, with $b \in \mathbb{R}^{m}, x \equiv[A]_{M P}^{-1} b$, with $x \in \mathbb{R}^{n}$ is the solution of the matrix equation with least norm. See [45]. 
2.2. Linear Dynamics of the Volume Preserving Hénon Map.

a) The Hénon map has fixed points

$$
f\left(x_{ \pm}, x_{ \pm}, x_{ \pm}\right)=\left(\begin{array}{c}
x_{ \pm} \\
x_{ \pm} \\
x_{ \pm}
\end{array}\right),
$$

where

$$
x_{ \pm}=\frac{-\tau}{2} \pm \frac{\sqrt{\tau^{2}-4 \alpha}}{2} .
$$

b) The jacobian is

$$
D f(x, y, z)=\left(\begin{array}{ccc}
2 a x+b y+\tau & 2 c y+b x & 1 \\
1 & 0 & 0 \\
0 & 1 & 0
\end{array}\right) .
$$

c) As determinant of the differential is identically one, generically we will have either; three real eigenvalues, or a complex conjugate pair of eigenvalues with the third real. Since the product of the three must be 1, we have either a two dimensional stable eigenspace, or a two dimensional unstable eigenspace.

3. 2-D Parametrization Method. In this section we adapt the theory of [11, 12 ] as needed for the current work. Namely, we develop the parameterization method for two dimensional stable and unstable manifolds of the hyperbolic fixed points of volume preserving Hénon map. There is so little difference between the computation of the two dimensional stable and unstable manifolds that we present only the stable case for the sake of brevity.

3.1. General set-up. Suppose $p \in \mathbb{R}^{3}$ is a hyperbolic fixed point with two dimensional stable eigenspace. In the work that follows we assume that the stable eigenvalues of $D f(p)$ are a complex conjugate pair. The computations for distinct real eigenvalues are similar. Suppose that the stable eigenvalues of $D f(p)$ are $\lambda$ and $\bar{\lambda}$, with $\lambda=\mu+i \nu$ and $\nu>0$. For each $\lambda \in \mathbb{C}$, we define the $2 \times 2$ matrix

$$
E_{\lambda}=\left(\begin{array}{cc}
\mu & -\nu \\
\nu & \mu
\end{array}\right) .
$$

We gather the properties of $E_{\lambda}$ in the following simple result.

Lemma 3.1. If $|\lambda|<1$ then the matrix $E_{\lambda}$ satisfies the following.

a) $E_{\lambda}^{n}=E_{\lambda^{n}}$, for all $n \in \mathbb{Z}$.

b) $E_{\lambda}\left(B_{\rho}(0)\right) \subset B_{\rho}(0)$.

c) $s^{\prime}+i t^{\prime}=\lambda(s+i t) \in \mathbb{C}$ if and only if

$$
\left(\begin{array}{c}
s^{\prime} \\
t^{\prime}
\end{array}\right)=E_{\lambda}\left(\begin{array}{c}
s \\
t
\end{array}\right) .
$$

d) $\lim _{n \rightarrow \infty} E_{\lambda}^{n}(s, t)=0$, for all $(s, t) \in \mathbb{R}^{2}$.

e) $E_{\lambda}$ is similar to $\left.D f(p)\right|_{\mathbb{E}^{s}}$. 
Now we develop the invariance equation which will allow us to compute a parameterization of the stable manifold. Let $\rho>0$, and define the domain $D^{s}=B_{\rho}(0) \subset \mathbb{R}^{2}$. We want to find $P_{s}: D^{s} \rightarrow \mathbb{R}^{3}$ so that

$$
f \circ P_{s}(s, t)=P_{s} \circ E_{\lambda}(s, t)
$$

for all $(s, t) \in D^{s}$. This is because if we assume that $P_{s}: D^{s} \subset \mathbb{R}^{2} \rightarrow \mathbb{R}^{3}$ is a smooth injection solving the Equation (3.2) on $D^{s}$, then $P_{s}\left(D^{s}\right)=W_{l o c}^{s}(p)$. To see this, we make three observations.

The first is that evaluation of the invariance equation at $(s, t)=(0,0)$ requires

$$
f \circ P_{s}(0,0)=P_{s} \circ E_{\lambda}(0,0)=P_{s}(0,0)
$$

as the linear map $E_{\lambda}$ fixes the origin. Then $P_{s}(0,0)$ is a fixed point of $f$, and of course we fix $P_{s}(0,0)=p$.

Next consider the first order constraint obtained by taking the derivative of Equation $(3.2)$ at $(s, t)=(0,0)$ :

$$
D f(p) D P(0,0)=D P(0,0) E_{\lambda} .
$$

The equation above implies that, if $v \in \mathbb{R}^{2}$ is an eigenvector of $E_{\lambda}$, then $D P(0,0) v$ is an eigenvector of the matrix $D f(p)$ with the same eigenvalue. In our situation, the matrix $E_{\lambda}$ has an eigenvector of the form $(1,-i)$ with eigenvalue $\lambda$. So, $D P(0,0)$ must be chosen so that $D P(0,0)(1,-i)$ is an eigenvector with eigenvalue $\lambda$. In particular, $P_{s}$ is tangent to the stable eigenspace of $D f(p)$.

Lastly, if $\left(s_{0}, t_{0}\right) \in D^{s} \subset \mathbb{R}^{2}$ with $\left(s_{0}, t_{0}\right) \neq 0$, and $q=P_{s}\left(s_{0}, t_{0}\right)$, we compute the trajectory of $q$ in phase space using the invariance equation. We have that

$$
f(q)=f \circ P_{s}\left(s_{0}, t_{0}\right)=P_{s} \circ E_{\lambda}\left(s_{0}, t_{0}\right) .
$$

Iterating this expression gives

$$
f^{n}(q)=f^{n} \circ P_{s}\left(s_{0}, t_{0}\right)=P_{s} \circ E_{\lambda}^{n}\left(s_{0}, t_{0}\right)
$$

so that

$$
\lim _{n \rightarrow \infty} f^{n}(q)=p
$$

as

$$
\lim _{n \rightarrow \infty} E_{\lambda}^{n}\left(s_{0}, t_{0}\right)=0
$$

and $P_{s}$ is continuous.

Then $P_{s}\left(D^{s}\right)$ is an embedded disk containing $p$, tangent to the stable eigenspace of $D f(p)$, and having that if $q \in P_{s}\left(D^{s}\right)$, then $f^{n}(q) \rightarrow p$ as $n \rightarrow \infty$. It follows that $P_{s}\left(D^{s}\right)$ is a local stable manifold of $p$ by definition. The situation is illustrated in Figure 3.1. 


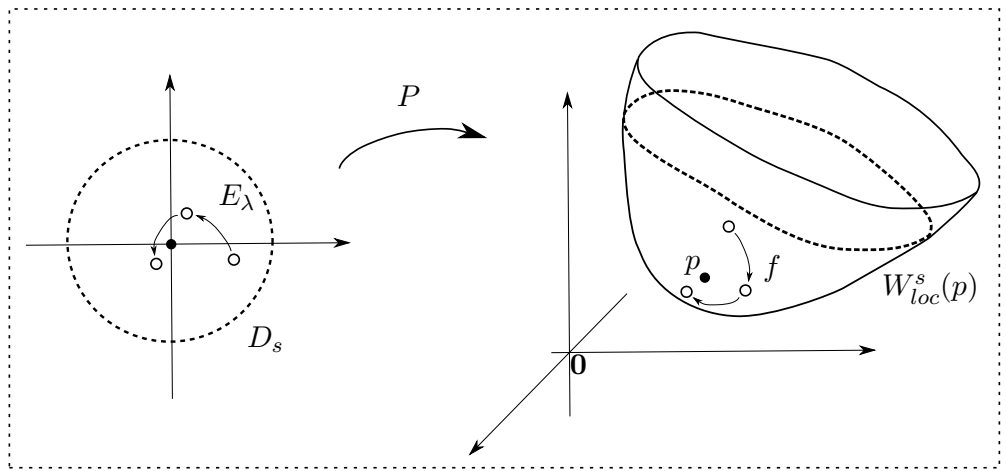

FIG. 3.1. Schematic of correspondence between the dynamics in the phase and parameter spaces, and motivation of the invariance equation.

In order to solve Equation (3.2) we assume that $P_{s}$ admits a power series representation. Because we are considering the case of complex conjugate eigenvalues, it is a useful ansatz to consider that the power series for $P_{s}$ is of the form

$$
P_{s}(s, t)=Q(s+i t)
$$

where $Q$ is a complex series of the form

$$
Q(z)=\sum_{n=0}^{\infty} \sum_{m=0}^{\infty} z^{m} \bar{z}^{n} \mathbf{q}_{m n}
$$

and $\mathbf{q}_{m n} \in \mathbb{C}^{3}$. Further, we assume that

$$
\overline{\mathbf{q}_{m n}}=\mathbf{q}_{n m} .
$$

Under this assumption, if $(s, t) \in \mathbb{R}^{2}$ then the series

$$
P_{s}(s, t)=\sum_{n=0}^{\infty} \sum_{m=0}^{\infty}(s+i t)^{m}(s-i t)^{n} \mathbf{q}_{m n}
$$

defines a map $P_{s}: \mathbb{R}^{2} \rightarrow \mathbb{R}^{3}$. We say that a power series whose coefficients satisfy Equation (3.7), is a complex conjugate series. Using Lemma 3.1, we have that

$$
P_{s} \circ E_{\lambda}(s, t)=Q(\lambda(s+i t)),
$$

and, in general, $P_{s} \circ E_{\lambda}^{n}(s, t)=Q\left(\lambda^{n}(s+i t)\right)$.

In order to determine the linear terms of $P_{s}$ we impose the constraints given by Equations (3.3) and (3.4). Then denote $\mathbf{q}_{m n}=\left(a_{m n}, b_{m n}, c_{m n}\right)$, and since $P_{s}(0,0)=p$ we have that

$$
\left(\begin{array}{l}
a_{00} \\
b_{00} \\
c_{00}
\end{array}\right)=p .
$$


To fix the next terms, note that the partial derivatives of complex conjugate series are given by

$$
\frac{\partial}{\partial s} P_{s}(s, t)=\sum_{n=0}^{\infty} \sum_{m=0}^{\infty}(s+i t)^{m}(s-i t)^{n}\left[(m+1) \mathbf{q}_{(m+1) n}+(n+1) \mathbf{q}_{m(n+1)}\right]
$$

and

$$
\frac{\partial}{\partial t} P_{s}(s, t)=\sum_{n=0}^{\infty} \sum_{m=0}^{\infty}(s+i t)^{m}(s-i t)^{n} i\left[(m+1) \mathbf{q}_{(m+1) n}-(n+1) \mathbf{q}_{m(n+1)}\right] .
$$

Evaluating the partial derivatives at $s=t=0$ results in

$$
\frac{\partial}{\partial s} P(0,0)=\left(\begin{array}{l}
a_{10} \\
b_{10} \\
c_{10}
\end{array}\right)+\left(\begin{array}{c}
a_{01} \\
b_{01} \\
c_{01}
\end{array}\right)=2 \operatorname{Re}\left(\begin{array}{c}
a_{10} \\
b_{10} \\
c_{10}
\end{array}\right)
$$

and

$$
\frac{\partial}{\partial t} P(0,0)=i\left(\begin{array}{c}
a_{10} \\
b_{10} \\
c_{10}
\end{array}\right)-i\left(\begin{array}{c}
a_{01} \\
b_{01} \\
c_{01}
\end{array}\right)=-2 \operatorname{Im}\left(\begin{array}{c}
a_{10} \\
b_{10} \\
c_{10}
\end{array}\right)
$$

Combining this with Equation (3.4), we have that

$$
\operatorname{DP}(0,0)\left(\begin{array}{c}
1 \\
-i
\end{array}\right)=2\left(\begin{array}{l}
a_{10} \\
b_{10} \\
c_{10}
\end{array}\right) \text {. }
$$

Therefore, we can choose $\left(a_{10}, b_{10}, c_{10}\right)$ and $\left(a_{01}, b_{01}, c_{01}\right)$ to be any pair of complex conjugate vectors in the stable eigenspace of $D f(p)$. Since the eigenvectors are determined only up to their direction, the length of the eigenvectors is a free parameter in the determination of $P_{s}$.

3.2. Computational Newton Method and Formal Series. In this section we develop a formal Newton Method for the iterative solution of Equation (3.2).

DeFinition 3.1.

a) Let $\mathbb{P}_{m, n}$ be the vector space of all formal power series of $m$ real variables, taking coefficients in $\mathbb{R}^{n}$.

b) Let $\mathbb{P} \mathbb{C}_{m, n}$ be the space of formal power series of $m$ complex variables, taking coefficients in $\mathbb{C}^{n}$.

c) Let $\mathbb{P}_{m, n}^{c}$ be the subset of $\mathbb{P} \mathbb{C}_{m, n}$ having complex conjugate variables and coefficients.

Note that while a formal series need not converge except at zero, any truncation of a a formal series is just a polynomial. Note also that every $Q \in \mathbb{P}_{m, n}^{c}$ corresponds to a $P \in \mathbb{P}_{2 m, n}$ through the equation

$$
P\left(s_{1}, \ldots, s_{m}, t_{1}, \ldots, t_{m}\right)=Q\left(s_{1}+i t_{1}, \ldots, s_{m}+i t_{m}\right)
$$


For the purposes of this paper, the most useful spaces are $\mathbb{P}_{1,3}, \mathbb{P}_{1,3}^{c}, \mathbb{P}_{1,4}$, and certain of their subsets. Any $P \in \mathbb{P}_{1,3}^{c}$ can be expressed as in Equation (3.8).

Now define

$$
\mathbb{P}_{1,3}^{c, 0}=\left\{h \in \mathbb{P}_{1,3}^{c}: h(0)=0 \text { and } D h(0)=0\right\}
$$

Since we know the linear part of the parameterization, we define $P_{0} \in \mathbb{P}_{1,3}^{c}$ by

$$
P_{0}(s, t)=\left(\begin{array}{c}
a_{00} \\
b_{00} \\
c_{00}
\end{array}\right)+(s+i t)\left(\begin{array}{c}
a_{01} \\
b_{01} \\
c_{01}
\end{array}\right)+(s-i t)\left(\begin{array}{c}
a_{10} \\
b_{10} \\
c_{10}
\end{array}\right),
$$

where these coefficients were given explicitly in Subsection 3.1. Now,the problem is to compute $h \in \mathbb{P}_{1,3}^{c, 0}$ so that

$$
P(s, t)=P_{0}(s, t)+h(s, t) \quad \text { and } \quad f \circ P=P \circ E_{\lambda}
$$

(we momentarily suppress the subscript on $P_{s}$ ). To this end, define an operator $\Psi: \mathbb{P}_{1,3}^{c, 0} \rightarrow \mathbb{P}_{1,3}^{c, 0}$ by

$$
\Psi(h)=f \circ\left(P_{0}+h\right)-\left(P_{0}+h\right) \circ E_{\lambda} .
$$

We aim to solve the equation $\Psi(h)=0$, by iterating the Newton sequence

$$
h_{n+1}=h_{n}-\left[D \Psi\left(h_{n}\right)\right]^{-1} \circ \Psi\left(h_{n}\right) .
$$

If the sequence converges, then the $n$-th approximate parameterization is $P_{n}=$ $P_{0}+h_{n}$. At the moment the scheme just discussed is purely formal. In order to develop a practical numerical implementation of the Newton method we must explicitly determine:

- A series development of $\Psi(h) \in \mathbb{P}_{1,3}^{c, 0}$, in terms of the known coefficients of $h \in \mathbb{P}_{1,3}^{c, 0}$.

- The Fréchet derivative $D \Psi(h)$ as a formal series in $\mathbb{P}_{1,3}^{c, 0}$, depending on the known coefficients of $h$.

- Invert the derivative, to obtain a formal series expansion of $[D \Psi(h)]^{-1} \circ Q \in$ $\mathbb{P}_{1,3}^{c, 0}$ in terms of the known coefficients of the formal series of $h, Q \in \mathbb{P}_{1,3}^{c, 0}$.

- An appropriate $h_{0}$ from which to begin the Newton iteration.

3.3. Series Development of $\Psi(h)$. For an arbitrary formal series $h \in \mathbb{P}_{1,3}^{c, 0}$ we want to define a new formal series

$$
h^{\prime}=\Psi(h)=f \circ\left(P_{0}+h\right)-\left(P_{0}+h\right) \circ E_{\lambda} .
$$

In other words, we hope to express $\Psi$ as a function from $\mathbb{P}_{1,3}^{c}$ to $\mathbb{P}_{1,3}^{c}$. This requires the computation of both $f \circ\left(P_{0}+h\right)$ and $\left(P_{0}+h\right) \circ E_{\lambda}$ as formal power series. Note that the coefficients of $P_{0}$ have already been chosen so that $\Psi\left(P_{0}+h\right)$ is zero to second order.

$P_{0}+h$ is a formal series in $\mathbb{P}_{1,3}^{c}$, which we denote by $K=P_{0}+h$. Compute

$$
f \circ K(s, t)=\sum_{n=0}^{\infty} \sum_{m=0}^{\infty}(s+i t)^{m}(s-i t)^{n}\left(\begin{array}{c}
a_{m n}^{\prime} \\
b_{m n}^{\prime} \\
c_{m n}^{\prime}
\end{array}\right)=
$$




$$
\left(\begin{array}{c}
\alpha+\tau K_{1}(s, t)+K_{3}(s, t)+a K_{1}(s, t)^{2}+b K_{1}(s, t) K_{2}(s, t)+c K_{2}(s, t)^{2} \\
K_{1}(s, t) \\
K_{2}(s, t)
\end{array}\right)
$$

and evaluate at $s=t=0$ to obtain the constant term

$$
\left(\begin{array}{c}
a_{00}^{\prime} \\
b_{00}^{\prime} \\
c_{00}^{\prime}
\end{array}\right)=\left(\begin{array}{c}
\alpha+\tau a_{00}+c_{00}+a a_{00}^{2}+b a_{00} b_{00}+c b_{00}^{2} \\
a_{00} \\
b_{00}
\end{array}\right)
$$

Expanding the products using the convolution formula from Appendix A.1, and matching like powers gives the recurrence relations for the general term

$$
\left(\begin{array}{c}
a_{m n}^{\prime} \\
b_{m n}^{\prime} \\
c_{m n}^{\prime}
\end{array}\right)=\left(\begin{array}{c}
\tau a_{m n}+c_{m n}+s_{m n} \\
a_{m n} \\
b_{m n}
\end{array}\right)
$$

where

$$
s_{m n}=\sum_{i=0}^{n} \sum_{j=0}^{m} a a_{(m-j)(n-i)} a_{j i}+b a_{(m-j)(n-i)} b_{j i}+c b_{(m-j)(n-i)} b_{j i} .
$$

The second term is $K \circ E_{\lambda}$. This term is already diagonalized over $\mathbb{P}_{1,3}^{c}$, as shown in Equation (3.9). Then, upon matching like powers we obtain that the coefficients for the $K \circ E_{\lambda} \in \mathbb{P}_{1,3}^{c}$ are

$$
\left(\begin{array}{c}
\hat{a}_{m n} \\
\hat{b}_{m n} \\
\hat{c}_{m n}
\end{array}\right)=\lambda^{m} \bar{\lambda}^{n}\left(\begin{array}{c}
a_{m n} \\
b_{m n} \\
c_{m n}
\end{array}\right)
$$

The simplicity of this formula is the reason for the choice of complex conjugate variables for the formal series of $\mathbb{P}_{1,3}^{c}$.

Letting $\left\{\tilde{a}_{m n}, \tilde{b}_{m n}, \tilde{c}_{m n}\right\}$ denote the general coefficient for $\Psi(P)$ and combining Equations (3.12) and (3.13) gives

$$
\left(\begin{array}{c}
\tilde{a}_{m n} \\
\tilde{b}_{m n} \\
\tilde{c}_{m n}
\end{array}\right)=\left(\begin{array}{c}
a_{m n}^{\prime}-\hat{a}_{m n} \\
b_{m n}^{\prime}-\hat{b}_{m n} \\
c_{m n}^{\prime}-\hat{c}_{m n}
\end{array}\right)
$$

Using these relations we can recursively compute the formal series for $\Psi(h)$ up to any desired finite order.

3.4. Fréchet Derivative of $\Psi$. Consideration of the difference

$$
\Psi(h+R)-\Psi(h) \quad h, R \in \mathbb{P}_{2,3}^{0}
$$

and a standard computation show that the Fréchet derivative is of $\Psi$ at $h \in \mathbb{P}_{1,3}^{c, 0}$ is

$$
[D \Psi(h)](R)=D f\left(P_{0}+h\right) R-R \circ E_{\lambda} .
$$


We require an explicit series representation of this derivative in terms of the known coefficients of the series $K=P_{0}+h$ and $R$. Since the linear term is already diagonalized (see Equation (3.9)) we need only compute the series expansion of $D f\left(P_{0}+h\right) R$.

Denote the unknown series by

$$
[D f(K) R](s, t)=\sum_{n=0}^{\infty} \sum_{m=0}^{\infty}(s+i t)^{m}(s-i t)^{n}\left(\begin{array}{c}
k_{m n}^{1} \\
k_{m n}^{2} \\
k_{m n}^{3}
\end{array}\right) .
$$

Using the known analytic expression for $D f$ (see Remark b) and the convolution formula from Appendix A.1, we expand the left hand side of Equation (3.16), and match like powers of the complex conjugate variables to obtain the recurrence relations

$$
\left(\begin{array}{c}
k_{m n} \\
k_{m n} \\
k_{m n}
\end{array}\right)=\left(\begin{array}{c}
r_{m n}^{3}+\tau r_{m n}^{1}+s_{m n}^{\prime} \\
r_{m n}^{1} \\
r_{m n}^{2}
\end{array}\right)
$$

where

$s_{m n}^{\prime}=\sum_{i=0}^{n} \sum_{j=0}^{m}\left[2 a a_{(m-j)(n-i)} r_{j i}^{1}+b b_{(m-j)(n-i)} r_{j i}^{1}+2 c b_{(m-j)(n-i)} r_{j i}^{2}+b a_{(m-j)(n-i)} r_{i j}^{2}\right]$

Combining Equation (3.17) with Equation (3.9) gives the general coefficients of $[D \Psi(K)](R)$ to any desired finite order.

3.5. Inversion of the derivative. Given fixed formal series $g$ and $h$ in $\mathbb{P}_{1,3}^{c, 0}$ we must compute the formal series expansion of

$$
r=[D \Psi(h)]^{-1} \circ g,
$$

which is equivalent to solving the equation

$$
D \Psi(h) \circ r=\left[D f\left(P_{0}+h\right) \circ r-r \circ E_{\lambda}\right]=g .
$$

Expand Equation (3.18) using Equations (3.9) and (3.17) and match like terms to obtain

$$
\left(\begin{array}{c}
r_{m n}^{3}+\tau r_{m n}^{1}-\lambda^{m} \bar{\lambda}^{n} r_{m n}^{1}+S_{m n} \\
r_{m n}^{1}-\lambda^{m} \bar{\lambda}^{n} r_{m n}^{2} \\
r_{m n}^{2}-\lambda^{m} \bar{\lambda}^{n} r_{m n}^{3}
\end{array}\right)=\left(\begin{array}{c}
g_{m n}^{1} \\
g_{m n}^{2} \\
g_{m n}^{3}
\end{array}\right)
$$

where

$S_{m n}=\sum_{i=0}^{n} \sum_{j=0}^{m}\left[2 a a_{(m-j)(n-i)} r_{j i}^{1}+b b_{(m-j)(n-i)} r_{j i}^{1}+2 c b_{(m-j)(n-i)} r_{j i}^{2}+b a_{(m-j)(n-i)} r_{i j}^{2}\right]$,

From this we isolate the $r_{m n}^{\ell}$ terms, and obtain that the matrix equation for the coefficients of $r$ are given by

$$
\left(\begin{array}{ccc}
-\lambda^{m} \bar{\lambda}^{n}+2 a a_{00}+b b_{00}+\tau & 2 c b_{00}+b a_{00} & 1 \\
1 & -\lambda^{m} \bar{\lambda}^{n} & 0 \\
0 & 1 & -\lambda^{m} \bar{\lambda}^{n}
\end{array}\right)\left(\begin{array}{c}
r_{m n}^{1} \\
r_{m n}^{2} \\
r_{m n}^{3}
\end{array}\right)=\left(\begin{array}{c}
g_{m n}^{1}-\tilde{s}_{m n} \\
g_{m n}^{2} \\
g_{m n}^{3}
\end{array}\right)
$$


where the

$\tilde{s}_{m n}=\sum_{i=0}^{n-1} \sum_{j=0}^{m-1}\left[2 a a_{(m-j)(n-i)} r_{j i}^{1}+b b_{(m-j)(n-i)} r_{j i}^{1}+2 c b_{(m-j)(n-i)} r_{j i}^{2}+b a_{(m-j)(n-i)} r_{i j}^{2}\right]$, depend only on lower order terms.

This sequence of infinitely many systems of three equations in three unknowns implicitly define the coefficients of $r$. The matrix term $A_{m n}$ is seen to be

$$
A_{m n}=\operatorname{Df}\left(p_{0}\right)-\lambda^{m} \bar{\lambda}^{n} I
$$

and we can invert as long as $\lambda^{m} \bar{\lambda}^{n}$ is not an eigenvalue of $D f\left(p_{0}\right)$. Since $\lambda, \bar{\lambda}$ are eigenvalues of $A_{m n}$, the system is invertible as long as

$$
\lambda^{m} \bar{\lambda}^{n} \neq \lambda
$$

and

$$
\lambda^{m} \bar{\lambda}^{n} \neq \bar{\lambda}
$$

These conditions are met whenever $m$ or $n$ are greater than one, as we are considering the case $|\lambda|<1$. Then we can solve the matrix equations except in the cases $m=n=0, m=1, n=0$ and $m=0, n=1$ (the cases for which the coefficients are already know). Solving the matrix equation gives a numerical procedure for computing the coefficients of $r$ to whatever finite order we like.

\subsection{Other remarks.}

a) We choose initial condition $h_{0}=0$ to begin the iteration. Note that while $\Psi\left(h_{0}\right) \in \mathbb{P}_{1,3}^{c, 0}$, it is not the case that $\Psi(0)=0$. This can be seen by considering $\Psi(0)=f \circ P_{0}-P_{0} \circ E_{\lambda}$ and using the explicit form of $P_{0}, E_{\lambda}$ and $f$.

b) The computations above are purely formal. Nevertheless, it is shown in [12], that such Newton schemes converges on the space of parameterizations analytic on some small enough disk $B_{r}(0)$, provided that $f$ and it's inverse are analytic, and provided that the non-resonance conditions given by Equations (3.20), and (3.21) are satisfied. Furthermore, if $f$ and its inverse are entire (as is the case for polynomial maps with polynomial inverses) the parameterizations are entire. Then we are assured in practice that our algorithms will converge [12].

c) The solution of Equation (3.2) is determined only up to the choice of the length of the eigenvectors. This is the only indeterminacy in the problem, as once we choose eigenvectors the higher order terms are uniquely determined by Equation (3.19).

d) The formal series for the two dimensional unstable manifold is computed in exactly the same way, the difference being that the $\lambda$ and $\bar{\lambda}$ are complex conjugate eigenvalues of $D f(p)$ having norm greater than one. From now on, we will write $P_{s}$ and $P_{u}$ for the parameterizations of the two dimensional stable and unstable manifolds respectively. 
e) The computations in this section are specific to the volume preserving Hénon family, as we have used the specific form of the map $f$ throughout. In principle the method can be applied to any polynomial map $f$ in a similar way. For a general analytic function $f$ one would proceed by expanding as a Taylor series to high order, and then computing the needed compositions and derivatives.

\section{Power series approximation of Heteroclinic Arcs.}

4.1. Set up of a functional equation, and Newton Scheme. Let $p_{0}$ and $p_{1}$ be hyperbolic fixed points of $f$ having two dimensional stable and unstable manifolds $W^{u}\left(p_{0}\right)$ and $W^{s}\left(p_{1}\right)$, which intersect transversally at a point $q$. Suppose that $P_{u}$ : $D_{u} \rightarrow \mathbb{R}^{3}$ and $P_{s}: D_{s} \rightarrow \mathbb{R}^{3}$ are parameterizations of the stable manifold of $p_{1}$ and the unstable manifold of $p_{0}$ respectively. While it is possible that

$$
P_{u}\left(D_{u}\right) \cap P_{s}\left(D_{s}\right)=W_{l o c}^{u}\left(p_{0}\right) \cap W_{l o c}^{s}\left(p_{1}\right)=\emptyset,
$$

it is the case that

$$
q \in f^{k_{u}}\left(W_{l o c}^{u}\left(p_{0}\right)\right) \cap f^{-k_{s}}\left(W_{l o c}^{s}\left(p_{1}\right)\right),
$$

for some $k_{u}, k_{s} \in \mathbb{N}$. Then there are $x_{0}^{u} \in D_{u}$ and $x_{0}^{s} \in D_{s}$ so that

$$
f^{k_{u}} \circ P_{u}\left(x_{0}^{u}\right)-f^{-k_{s}} \circ P_{s}\left(x_{0}^{s}\right)=0 .
$$

By the transversality assumption, the intersection in Equation (4.1) is locally a one dimensional arc $\tilde{\gamma} \subset W^{u}\left(p_{0}\right) \cap W^{s}\left(p_{1}\right)$ through $q$. Pulling $\tilde{\gamma}$ back to parameter space, there are $\operatorname{arcs} \gamma^{u} \subset D_{u}$ and $\gamma^{s} \subset D_{s}$ having $\gamma^{u}(0)=x_{0}^{u}, \gamma^{s}(0)=x_{0}^{s}$, and such that

$$
f^{k_{u}} \circ P_{u} \circ \gamma^{u}-f^{-k_{s}} \circ P_{s} \circ \gamma^{s}=0 .
$$

Define the product curve $\gamma:[-\tau, \tau] \subset \mathbb{R} \rightarrow D_{u} \times D_{s} \subset \mathbb{R}^{4}$ by $\gamma=\left(\gamma^{u}, \gamma^{s}\right)$ with $\gamma(0)=$ $\left(x_{0}^{u}, x_{0}^{s}\right)$. The situation is illustrated in Figure 4.1 (with $f$ and $f^{-1}$ compositions suppressed).

The linear term of $\gamma$ can be determined by considering the tangent vector $\gamma^{\prime}(0)$. To this end it is useful to define the function $F: D_{u} \times D_{s} \rightarrow \mathbb{R}^{3}$ by

$$
F\left(x^{u}, x^{s}\right) \equiv f^{k_{u}} \circ P_{u}\left(x^{u}\right)-f^{-k_{s}} \circ P_{s}\left(x^{s}\right),
$$

with $\left(x^{s}, x^{s}\right) \in D_{u} \times D_{s}$. Of course

$$
F\left(x_{u}, x_{s}\right)=F[\gamma(0)]=0 .
$$

Recall from differential geometry that whenever a smooth arc $\gamma$ is defined as the zero set of the smooth function $F$, we have that

$$
\gamma^{\prime}(0) \in \operatorname{ker}\left(D F\left(x_{0}^{u}, x_{0}^{s}\right)\right) .
$$

(See section 1.4 of [19]). We note for future reference that 


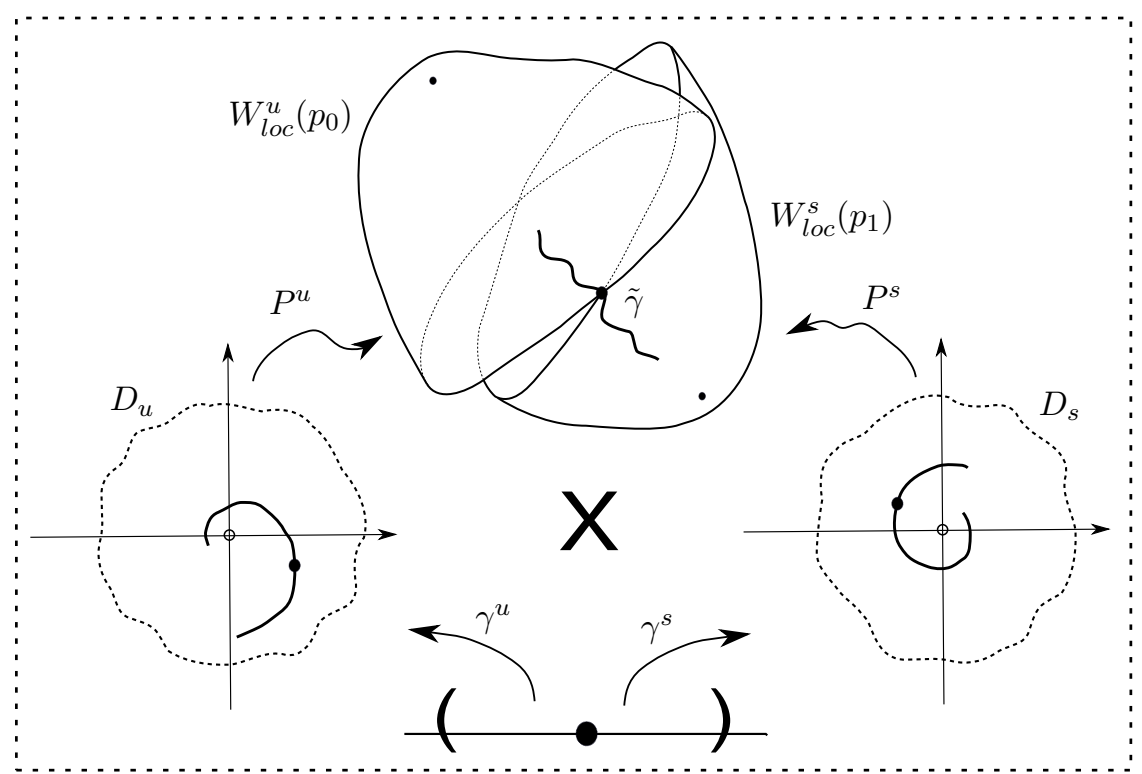

FIG. 4.1. Schematic motivation of the invariance equation for $\gamma$.

$$
D F\left(x_{0}^{u}, x_{0}^{s}\right)=\left[D F_{1} \mid D F_{2}\right]
$$

with

$$
D F_{1}=\left(\begin{array}{cc}
\frac{\partial}{\partial u_{1}}\left[f^{k_{u}} \circ P_{u}\left(u_{1}^{0}, u_{2}^{0}\right)\right]_{1} & \frac{\partial}{\partial u_{2}}\left[f^{k_{u}} \circ P_{u}\left(u_{1}^{0}, u_{2}^{0}\right)\right]_{1} \\
\frac{\partial}{\partial u_{1}}\left[f^{k_{u}} \circ P_{u}\left(u_{1}^{0}, u_{2}^{0}\right)\right]_{2} & \frac{\partial}{\partial u_{2}}\left[f^{k_{u}} \circ P_{u}\left(u_{1}^{0}, u_{2}^{0}\right)\right]_{2} \\
\frac{\partial}{\partial u_{1}}\left[f^{k_{u}} \circ P_{u}\left(u_{1}^{0}, u_{2}^{0}\right)\right]_{3} & \frac{\partial}{\partial u_{2}}\left[f^{k_{u}} \circ P_{u}\left(u_{1}^{0}, u_{2}^{0}\right)\right]_{3}
\end{array}\right)
$$

and

$$
D F_{2}=\left(\begin{array}{cc}
\frac{\partial}{\partial s_{1}}\left[f^{-k_{s}} \circ P_{s}\left(s_{1}^{0}, s_{2}^{0}\right)\right]_{1} & \frac{\partial}{\partial s_{2}}\left[f^{-k_{s}} \circ P_{s}\left(s_{1}^{0}, s_{2}^{0}\right)\right]_{1} \\
\frac{\partial}{\partial s_{1}}\left[f^{-k_{s}} \circ P_{s}\left(s_{1}^{0}, s_{2}^{0}\right)\right]_{2} & \frac{\partial}{\partial s_{2}}\left[f^{-k_{s}} \circ P_{s}\left(s_{1}^{0}, s_{2}^{0}\right)\right]_{2} \\
\frac{\partial}{\partial s_{1}}\left[f^{-k_{s}} \circ P_{s}\left(s_{1}^{0}, s_{2}^{0}\right)\right]_{3} & \frac{\partial}{\partial s_{2}}\left[f^{-k_{s}} \circ P_{s}\left(s_{1}^{0}, s_{2}^{0}\right)\right]_{3}
\end{array}\right)
$$

Here we have let $x^{u}=\left(u_{1}, u_{2}\right), x^{s}=\left(s_{1}, s_{2}\right), x_{0}^{u}=\left(u_{1}^{0}, u_{2}^{0}\right)$ and $x_{0}^{s}=\left(s_{1}^{0}, s_{2}^{0}\right)$.

Suppose the kernel of $\operatorname{DF}\left(x_{0}^{u}, x_{0}^{s}\right)$ is one-dimensional, and choose a vector $0 \neq$ $v \in \operatorname{ker}\left(D F\left(x_{0}^{u}, x_{0}^{s}\right)\right.$-where the length of $v$ is arbitrary (this can be done numerically using the singular value decomposition as discussed in Section 2.1). Then the linear approximation to $\gamma$ is

$$
\gamma_{0}(\theta)=\left(x_{0}^{u}, x_{0}^{s}\right)+\theta v
$$

Now we will compute the high order terms for $\gamma$, based on the fact that $\gamma$ solves Equation (4.2). As in the case of the two dimensional stable and unstable manifolds, 
we solve the functional equation using a Newton method on a space of formal series. Begin by assuming that $\gamma(\theta)$ can be expressed as a power series

$$
\gamma(\theta)=\left(\begin{array}{c}
\gamma^{u}(\theta) \\
\gamma^{s}(\theta)
\end{array}\right)=\sum_{j=0}^{\infty} \theta^{j}\left(\begin{array}{c}
\alpha_{j} \\
\beta_{j} \\
\hat{\alpha}_{j} \\
\hat{\beta}_{j}
\end{array}\right)
$$

with $\left(\alpha_{0}, \beta_{0}, \hat{\alpha}_{0}, \hat{\beta}_{0}\right)=\left(x_{0}^{u}, x_{0}^{s}\right)$, and $\left(\alpha_{1}, \beta_{1}, \hat{\alpha}_{1}, \hat{\beta}_{1}\right)=v$. Note that all series of the form given in Equation (4.8) are elements of $\mathbb{P}_{1,4}$, and that $\gamma_{0} \in \mathbb{P}_{1,4}$.

Let

$$
\mathbb{P}_{1,4}^{0}=\left\{h \in \mathbb{P}_{1,4}: h(0)=0 \quad \text { and } \quad h^{\prime}(0)=0\right\}
$$

and

$$
\mathbb{P}_{1,3}^{0}=\left\{g \in \mathbb{P}_{1,3}: g(0)=0 \quad \text { and } \quad g^{\prime}(0)=0\right\} .
$$

For $h \in \mathbb{P}_{1,4}^{0}$, we write $h=\left(h^{u}, h^{s}\right)$.

This discussion motivates the definition the nonlinear operator $\Phi: \mathbb{P}_{1,4}^{0} \rightarrow \mathbb{P}_{1,3}^{0}$ by

$$
\Phi(h) \equiv f^{k_{u}} \circ P_{u} \circ\left(\gamma_{0}^{u}+h^{u}\right)-f^{-k_{s}} \circ P_{s} \circ\left(\gamma_{0}^{s}+h^{s}\right),
$$

where $\Phi$ is zero to second order in $\theta$ due to the definition of $\gamma_{0}$. To compute a zero of $\Phi$, we iterate the sequence

$$
h_{n+1}=h_{n}-\left[D \Phi\left(h_{n}\right)\right]^{-1} \circ \Phi\left(h_{n}\right) .
$$

Then $\gamma_{n}=\gamma_{0}+h_{n}$ is the $n$-th approximation to $\gamma$. As before, this formalism requires computation of:

- A formal series for $\Phi(h)$, given any formal series $h$

- The Fréchet derivative of $\Phi$ with respect to $h$.

- A formal series expansion for $[D \Phi(h)]^{-1} \circ g$, when $h$ and $g$ are known formal series.

- An appropriate starting function from which to begin the Newton iteration.

4.2. Composition of $\Phi$ and $h$. First consider the expressions $f^{k_{u},-k_{s}} \circ P_{u, s}$. The recursion relations for $f \circ Q$ where $Q \in \mathbb{P}_{1,3}^{c}$, are given by Equation (3.14). Then if $P_{u}$ is the series expansion of the unstable manifold we use these recurrence relations to compute the coefficients of $P^{1}=f \circ P_{u}$.

Now $P^{1} \in \mathbb{P}_{1,3}^{c}$ and we can compute the coefficients of

$$
P^{2}=f \circ P^{1}=f\left[f \circ P_{u}\right]=f^{2} \circ P_{u},
$$

using the same recurrence relations. By iterating we compute the formal series for $f^{k_{u}} \circ P_{u}$ for any $k_{u} \in \mathbb{N}$ (and similar computations for $f^{-k_{s}} \circ P_{s}$ ).

In this section we denote the series so obtained by

$$
f^{k_{u}} \circ P_{u}(s, t)=\sum_{n=0}^{\infty} \sum_{m=0}^{\infty}(s+i t)^{m}(s-i t)^{n}\left(\begin{array}{c}
a_{m n}^{1} \\
a_{m n}^{2} \\
a_{m n}^{3}
\end{array}\right)
$$


and

$$
f^{-k_{s}} \circ P_{s}(s, t)=\sum_{n=0}^{\infty} \sum_{m=0}^{\infty}(s+i t)^{m}(s-i t)^{n}\left(\begin{array}{c}
\hat{a}_{m n}^{1} \\
\hat{a}_{m n}^{2} \\
\hat{a}_{m n}^{3 n}
\end{array}\right) .
$$

In order to complete the computation of $\Phi(h)$, we have to compute the further compositions $f^{k_{u}} \circ P_{u} \circ\left(\gamma_{0}^{u}+h^{u}\right)$ and $f^{-k_{s}} \circ P_{s} \circ\left(\gamma_{0}^{s}+h^{s}\right)$. The component equations are

$$
\begin{gathered}
{\left[f^{k_{u}} \circ P_{u} \circ\left(\gamma_{0}^{u}\right)\right]_{\ell}=} \\
\sum_{n=0}^{\infty} \sum_{m=0}^{\infty}\left[\sum_{j=0}^{\infty}\left(\alpha_{j}+i \beta_{j}\right) \theta^{j}\right]^{m}\left[\sum_{j=0}^{\infty}\left(\alpha_{j}-i \beta_{j}\right) \theta^{j}\right]^{n} a_{m n}^{\ell}=\sum_{j=0}^{\infty} A_{j}^{\ell} \theta^{j}
\end{gathered}
$$

and

$$
\begin{gathered}
{\left[f^{-k_{s}} \circ P_{s} \circ\left(\gamma_{0}^{s}\right)\right]_{\ell}=} \\
\sum_{n=0}^{\infty} \sum_{m=0}^{\infty}\left[\sum_{j=0}^{\infty}\left(\hat{\alpha}_{j}+i \hat{\beta}_{j}\right) \theta^{j}\right]^{m}\left[\sum_{j=0}^{\infty}\left(\hat{\alpha}_{j}-i \hat{\beta}_{j}\right) \theta^{j}\right]^{n} \hat{a}_{m n}^{\ell}=\sum_{j=0}^{\infty} \hat{A}_{j}^{\ell} \theta^{j}
\end{gathered}
$$

for $\ell=1,2,3$ and some $A_{j}^{\ell}, \hat{A}_{j}^{\ell}$ to be determined. Note however that these compositions have the form of the general compositions worked out in Appendix A.2, so that we can explicitly compute the coefficients $A_{j}^{1}, A_{j}^{2}, A_{j}^{3}, \hat{A}_{j}^{1}, \hat{A}_{j}^{2}$, and $\hat{A}_{j}^{3}$ using the method discussed there.

4.3. Fréchet Derivative of $\Phi$ With Respect to $h$. Let $r, h \in \mathbb{P}_{1,4}^{0}$. We denote these functions by $h=\left(h^{u}, h^{s}\right)$ and $r=\left(r^{u}, r^{s}\right)$. Define $\xi=\gamma_{0}+h$, with $\xi=\left(\xi^{u}, \xi^{s}\right)$. Considering the difference

$$
\Phi(h+r)-\Phi(h),
$$

and using Taylors Theorem to expand $f$ allows us to compute the Fréchet derivative

$$
[D \Phi(\gamma)](r)=D\left[f^{k_{u}} \circ P_{u}\left(\xi^{u}\right)\right]\left(r^{u}\right)-D\left[f^{-k_{s}} \circ P_{s}\left(\xi^{s}\right)\right]\left(r^{s}\right) .
$$

Applying the partial derivative formulas from Equations (3.10) and (3.11) to the series expansions for $f^{k_{u}} \circ P_{u}$ and $f^{-k_{s}} \circ P_{s}$ discussed in Section (4.2), we compute the matrix entries

$$
D\left[f^{k_{u}} \circ P_{u}\right]=\left(\begin{array}{cc}
\frac{\partial}{\partial u_{1}}\left[f^{k_{u}} \circ P_{u}\right]_{1} & \frac{\partial}{\partial u_{2}}\left[f^{k_{u}} \circ P_{u}\right]_{1} \\
\frac{\partial}{\partial u_{1}}\left[f^{k_{u}} \circ P_{u}\right]_{2} & \frac{\partial}{\partial u_{2}}\left[f^{k_{u}} \circ P_{u}\right]_{2} \\
\frac{\partial}{\partial u_{1}}\left[f^{k_{u}} \circ P_{u}\right]_{3} & \frac{\partial}{\partial u_{2}}\left[f^{k_{u}} \circ P_{u}\right]_{3}
\end{array}\right),
$$


and

$$
D\left[f^{-k_{s}} \circ P_{s}\right]=\left(\begin{array}{cc}
\frac{\partial}{\partial s_{1}}\left[f^{k_{s}} \circ P_{s}\right]_{1} & \frac{\partial}{\partial s_{2}}\left[f^{k_{s}} \circ P_{s}\right]_{1} \\
\frac{\partial}{\partial s_{1}}\left[f^{k_{s}} \circ P_{s}\right]_{2} & \frac{\partial}{\partial s_{2}}\left[f^{k_{s}} \circ P_{s}\right]_{2} \\
\frac{\partial}{\partial s_{1}}\left[f^{k_{s}} \circ P_{s}\right]_{3} & \frac{\partial}{\partial s_{2}}\left[f^{k_{s}} \circ P_{s}\right]_{3}
\end{array}\right) .
$$

Here each entry is a formal series in $\mathbb{P}_{2,3}$ with variables $x^{u}=\left(u_{1}, u_{2}\right)$ and $x^{s}=\left(s_{1}, s_{2}\right)$. We now apply the composition formula developed in Appendix A.2, and evaluate each entry at $\xi \in \mathbb{P}_{1,4}^{0}$ obtaining

$$
D\left[f^{k_{u}} \circ P_{u}\left(\xi^{u}\right)\right]=\sum_{j=0}^{\infty} \theta^{j}\left(\begin{array}{cc}
B_{j}^{1} & C_{j}^{1} \\
B_{j}^{2} & C_{j}^{2} \\
B_{j}^{3} & C_{j}^{3}
\end{array}\right),
$$

and

$$
D\left[f^{-k_{s}} \circ P_{s}\left(\xi^{s}\right)\right]=\sum_{j=0}^{\infty} \theta^{j}\left(\begin{array}{cc}
\hat{B}_{j}^{1} & \hat{C}_{j}^{1} \\
\hat{B}_{j}^{2} & \hat{C}_{j}^{2} \\
\hat{B}_{j}^{3} & \hat{C}_{j}^{3}
\end{array}\right) .
$$

Then the matrix series of the Fréchet derivative is

$$
[D \Phi(h)]=\sum_{j=0}^{\infty} \theta^{j}\left(\begin{array}{cccc}
B_{j}^{1} & C_{j}^{1} & -\hat{B}_{j}^{1} & -\hat{C}_{j}^{1} \\
B_{j}^{2} & C_{j}^{2} & -\hat{B}_{j}^{2} & -\hat{C}_{j}^{2} \\
B_{j}^{3} & C_{j}^{3} & -\hat{B}_{j}^{3} & -\hat{C}_{j}^{3}
\end{array}\right),
$$

and the composition $[D \Phi(h)] r$ for $r \in \mathbb{P}_{1,4}^{0}$ is computed by standard matrix multiplication, with the elements of both the vector $r$ and the matrix $D \Phi(h)$ power series of a single real variable. The multiplications are computed using the standard product formula. Note that the result

$$
[D \Phi(h)] r \in \mathbb{P}_{1,3}^{0} .
$$

4.4. Inversion of the Fréchet derivative. Now denote $g \in \mathbb{P}_{1,3}^{0}$ by

$$
g(\theta)=\sum_{j=0}^{\infty} \theta^{j}\left(\begin{array}{c}
g_{j}^{1} \\
g_{j}^{2} \\
g_{j}^{3}
\end{array}\right),
$$

with $g(0)=g^{\prime}(0)=0$. We must develop a formal series expansion for $h \in \mathbb{P}_{1,4}$ defined by

$$
r=[D \Phi(h)]^{-1} g .
$$


This is equivalent to finding an $r \in \mathbb{P}_{1,4}^{0}$ solving the equation

$$
[D \Phi(h)] r=g
$$

where $h$ and $g$ are fixed.

Using the series expansion of $D \Phi$ developed above, this is

$$
\left[\sum_{j=0}^{\infty} \theta^{j}\left(\begin{array}{cccc}
B_{j}^{1} & C_{j}^{1} & -\hat{B}_{j}^{1} & -\hat{C}_{j}^{1} \\
B_{j}^{2} & C_{j}^{2} & -\hat{B}_{j}^{2} & -\hat{C}_{j}^{2} \\
B_{j}^{3} & C_{j}^{3} & -\hat{B}_{j}^{3} & -\hat{C}_{j}^{3}
\end{array}\right)\right]\left[\sum_{j=0}^{\infty} \theta^{j}\left(\begin{array}{c}
\alpha_{j} \\
\beta_{j} \\
\hat{\alpha}_{j} \\
\hat{\beta}_{j}
\end{array}\right)\right]=\sum_{j=0}^{\infty} \theta^{j}\left(\begin{array}{c}
g_{j}^{1} \\
g_{j}^{2} \\
g_{j}^{3}
\end{array}\right) .
$$

Expanding a component of this equation using the convolution formula in Appendix A.1 gives

$$
\sum_{j=0}^{\infty} \sum_{k=0}^{j}\left[B_{k}^{\ell} \alpha_{j-k}+C_{k}^{\ell} \beta_{j-k}-\hat{B}_{k}^{\ell} \hat{\alpha}_{j-k}-\hat{C}_{k}^{\ell} \hat{\beta}_{j-k}\right] \theta^{j}=\sum_{j=0}^{\infty} g_{j}^{\ell} \theta^{j}
$$

for $\ell=1,2,3$. Matching like powers of $\theta^{j}$ and isolating the $j$-th terms of $r$ gives

$$
B_{0}^{\ell} \alpha_{j}+C_{0}^{\ell} \beta_{j}-\hat{B}_{0}^{\ell} \hat{\alpha}_{j}-\hat{C}_{0}^{\ell} \hat{\beta}_{j}=g_{j}^{\ell}-s_{j}^{\ell},
$$

for $\ell=1,2,3$, with $s_{j}^{\ell}$ defined by

$$
s_{j}^{\ell}=\sum_{k=1}^{j}\left[B_{k}^{\ell} \alpha_{j-k}+C_{k}^{\ell} \beta_{j-k}-\hat{B}_{k}^{\ell} \hat{\alpha}_{j-k}-\hat{C}_{k}^{\ell} \hat{\beta}_{j-k}\right] .
$$

Note that $s_{j}^{\ell}$ depend recursively on only lower order (hence already known) terms. Matrix equations for the coefficients of $r$ are

$$
\left(\begin{array}{cccc}
B_{0}^{1} & C_{0}^{1} & \hat{B}_{0}^{1} & \hat{C}_{0}^{1} \\
B_{0}^{2} & C_{0}^{2} & \hat{B}_{0}^{2} & \hat{C}_{0}^{2} \\
B_{0}^{3} & C_{0}^{3} & \hat{B}_{0}^{3} & \hat{C}_{0}^{3}
\end{array}\right)\left(\begin{array}{c}
\alpha_{j} \\
\beta_{j} \\
\hat{\alpha}_{j} \\
\hat{\beta}_{j}
\end{array}\right)=\left(\begin{array}{c}
g_{j}^{1}-s_{j}^{1} \\
g_{j}^{2}-s_{j}^{2} \\
g_{j}^{3}-s_{j}^{3}
\end{array}\right)
$$

or

$$
\mathfrak{D} r_{j}=g_{j}-s_{j}
$$

\subsection{Other Remarks.}

a) Once we have obtained series solution $\gamma=\left(\gamma^{u}, \gamma^{s}\right)$ of Equation (4.2), either of the curves $P_{u}\left(\gamma^{u}\right), P_{s}\left(\gamma^{s}\right) \in \mathbb{P}_{1,3}$ parameterize $\tilde{\gamma} \subset W^{u}\left(p_{0}\right) \cap W^{s}\left(p_{1}\right)$ in phase space.

b) We begin the Newton iteration with $h_{0}=0$. As before note that while $\Psi(0) \in \mathbb{P}_{1,4}^{0}$ it is not the case that $\Psi(0)=0$ (here 0 refers to the zero element of $\left.\mathbb{P}_{1,4}^{0}\right)$.

c) Unlike the Newton scheme developed in Section 3, the present scheme is general, as we have made no use of the specific form of $f$. What is required is that $f$ and its inverse are analytic, that $P_{s, u}$ are known, that the the recursion for $f \circ K$ and $f^{-1} \circ K$ are known, and that $q, k_{u}$, and $k_{s}$ are known. 
d) Since $\mathfrak{D}$ in Equation (4.14) is a $3 \times 4$ matrix, it cannot be invertible. Nevertheless, we obtain a solution of Equation (4.14) at each $j$ by employing the Moore-Penrose inverse of $\mathfrak{D}$ as discussed in Section 2.1. Then

$$
r_{j}=[\mathfrak{D}]_{M P}^{-1}\left(g_{j}-s_{j}\right) .
$$

uniquely determines the coefficients of $r$. Note that $\mathfrak{D}$ is independent of $j$, and the computation of the Moore-Penrose inverse of a single matrix is all that is required.

e) Moreover, we have that $\mathfrak{D}=D F\left(x_{0}^{u}, x_{0}^{s}\right)$, where $D F$ is the matrix from Equation (4.6). To see this simply evaluate Equation (4.12) at $\theta=0$ (using Equations (4.10), and (4.11)) and compare with the terms of Equation (4.6). Then we will learn when we attempt to fix $\gamma_{0}$ whether or not $\mathfrak{D}$ has one-dimensional kernel. It follows that if the initial curve $\gamma_{0}$ can computed numerically, then we can solve Equation (4.14), for any $h \in \mathbb{P}_{1,4}^{0}$ and $g \in \mathbb{P}_{1,3}^{0}$, and compute the coefficients of $r$ up to any fixed finite order.

f) The condition that $D F\left(x_{0}^{u}, x_{0}^{s}\right)$ in Equation (4.5) have one dimensional kernel is equivalent to the assumption that $W^{u}\left(p_{0}\right) \cap W^{s}\left(p_{1}\right)$ is transverse at $q$. Then in practice, we need not know a-priori that the intersection is transverse at $q$. We will learn wether the assumption of transversality holds, and wether $[D \Phi(h)]^{-1}$ is well defined, when we attempt to define $\gamma_{0}$ in Equation (4.5).

g) The fact that we have one degree of freedom in the Equation (4.14) at each $j$ is interpreted in terms of reparameterization of the speed along the $\operatorname{arc} \gamma$. Given a curve $\gamma=\gamma_{0}+h$ solving $\Phi(h)=0$ it is the case that any reparameterization $\gamma^{\prime}(\theta)=\gamma_{0}(\sigma(\theta))+h(\sigma(\theta))=\gamma_{0}+h^{\prime}$, with $\sigma: \mathbb{R} \rightarrow \mathbb{R}$ an increasing function of $\theta$, will also have $\Phi\left(h^{\prime}\right)=0$. The freedom in the choice of coefficient of $h$ at each power $j$ corresponds to the freedom in choosing the $j$-th Taylor coefficient of $\sigma$.

h) The under determinedness of Equation (4.14) and the freedom in choice of parameterization of $\gamma$ are related to the observation that $D \Phi(h)$ cannot be an isomorphism of $\mathbb{P}_{1,4}^{0}$ onto $\mathbb{P}_{1,3}^{0}$, and hence must have non-trivial kernel. In fact the since the kernel of $D \Phi(h)$ is parameterized by the family of increasing functions $\sigma$ from the previous remark, the kernel is not even finite dimensional. Solving Equation (4.13) is equivalent to computing a psudoinverse of an infinite dimensional linear operator having infinite dimensional kernel.

5. Numerical Domain of the Formal Series. Even though the parameterizations $P_{s, u}$ are entire (see Remark b in section 3), in practice we only ever compute a finite number of terms, and the truncated series only approximate the desired parameterizations. In order to make practical use of these truncated series, we have to determine parameter domains on which the approximations are reasonable. To this end, define the stable and unstable error functionals

$$
\text { Error }_{s}^{r}, \text { Error }_{u}^{r}: C^{0}\left(B_{r}(0) \subset \mathbb{R}^{2}, \mathbb{R}^{3}\right) \rightarrow \mathbb{R}
$$

by 


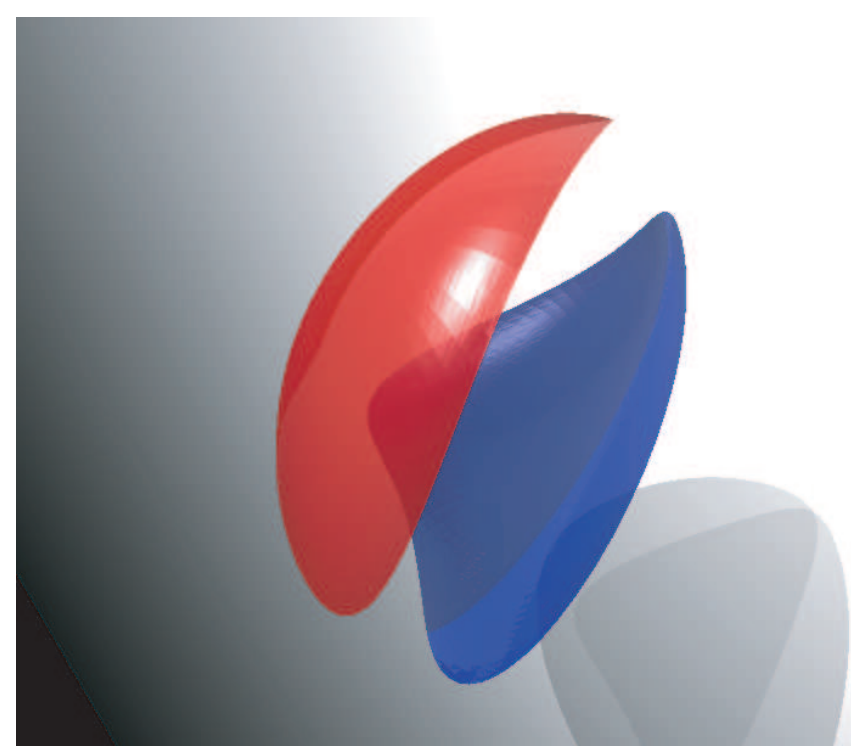

FIG. 5.1. $P_{s}^{N}\left(D_{s}\right)$ and $P_{u}^{N}\left(D_{u}\right)$, for $N=50$ and $\varepsilon=5 \times 10^{-15}$. Local unstable manifold shown in blue. Local stable in red.

$$
\operatorname{Error}_{u}^{r}(Q)=\sup _{(s, t) \in B_{r}(0)}\left\|\left(f \circ Q-Q \circ E_{\lambda_{u}}\right)(s, t)\right\|,
$$

and

$$
\operatorname{Error}_{s}^{r}(Q)=\sup _{(s, t) \in B_{r}(0)}\left\|\left(f \circ Q^{\prime}-Q^{\prime} \circ E_{\lambda_{s}}\right)(s, t)\right\|,
$$

where $Q, Q^{\prime} \in C^{0}\left(B_{r}(0) \subset \mathbb{R}^{2}, \mathbb{R}^{3}\right)$, and $r>0$ is free.

Let $P_{s, u}^{N}$ be the $N$-th order numerical approximations of the parameterizations $P_{s, u}$ obtained by iterating the Newton scheme discussed in Section 3, truncated at order $N$.

DEFINITION 5.1. We say that $r>0$ is an $\varepsilon$-numerical radius of convergence for $P_{s, u}^{N}$ if

$$
\operatorname{Error}_{s, u}^{r}\left(P_{s, u}^{N}\right)<\varepsilon
$$

on $B_{r}(0) \subset \mathbb{R}^{2}$.

Fix $\varepsilon>0$ and suppose that $r_{u, s}$ are $\varepsilon$-numerical radii of convergence for $P_{u, s}^{N}$. Define stable and unstable parameter disks $D_{u}=B_{r_{u}}(0)$ and $D_{s}=B_{r_{s}}(0)$. The numerical approximations of the local stable and unstable manifolds in phase space are given by

$$
W_{l o c}^{u} \approx P_{u}^{N}\left(D_{u}\right) \quad W_{l o c}^{s} \approx P_{s}^{N}\left(D_{s}\right),
$$

and $\varepsilon$ is a measure of how accurately $P_{s, u}^{N}$ approximate $P_{s, u}$ on $D_{s, u}$. 


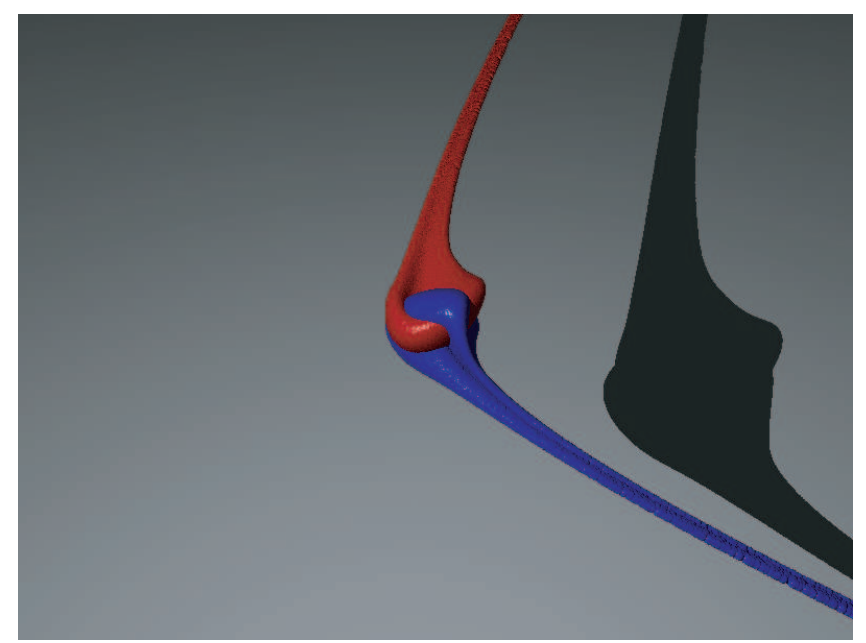

FIG. 5.2. Globalized Stable and Unstable manifolds. Obtained by iterating the local disks 75 times.

EXAMPLE 5.1. Take $a=0.44, b=0.21, c=0.35, \alpha=-0.25$, and $\tau=-0.3$. We truncate the power series $P_{s, u}$ at order $K=50$. Let $\varepsilon=5 \cdot 10^{-15}$. Evaluating the error functionals for various $r<1$ shows that $r_{s}=r_{u}=0.62$ is an $\varepsilon$-numerical radii of convergence for the series.

The resulting approximations of the stable and unstable manifolds are shown in Figure 5.1, with the stable manifold colored red and the unstable manifold colored blue. The surfaces shown in Figure 5.1 are obtained by triangulating $D_{s}$ and $D_{u}$ in the plane, and then lifting the triangulation into phase space using $P_{s}$ and $P_{u}$. Then it is the vertices of the Figures that satisfy the error conditions. The disks shown in Figure 5.1 require no iteration under the Hénon dynamics. Figure 5.2 is obtained by iterating the local disks 75 times each.

REMARKS 5.1.

- The polynomial approximations of the stable and unstable manifolds are valid on a large region of phase space. This is illustrated in Figure 5.1 by the fact that the local stable and unstable manifolds occupy much of the region between the fixed points.

- The parameterization method yields highly accurate polynomial approximations (in this case the order 50 polynomials are accurate to within roughly 23 multiples of double precision machine epsilon on $\left.D_{s, u}\right)$. Because the local approximations are so precise, it is possible to iterate a large number of times before the approximations diverge from the actual stable and unstable manifolds.

- Figure 5.2 illustrates the correct asymptotic behavior of the two dimensional manifolds, which must accumulate on the one dimensional stable and unstable manifolds by the $\lambda$-lemma.

- Since the parameterizations are not required to be graphs, the method allows us to follow folds of the manifolds. The method also gives accurate information about derivatives as we compute the series to high order with small remainder. 


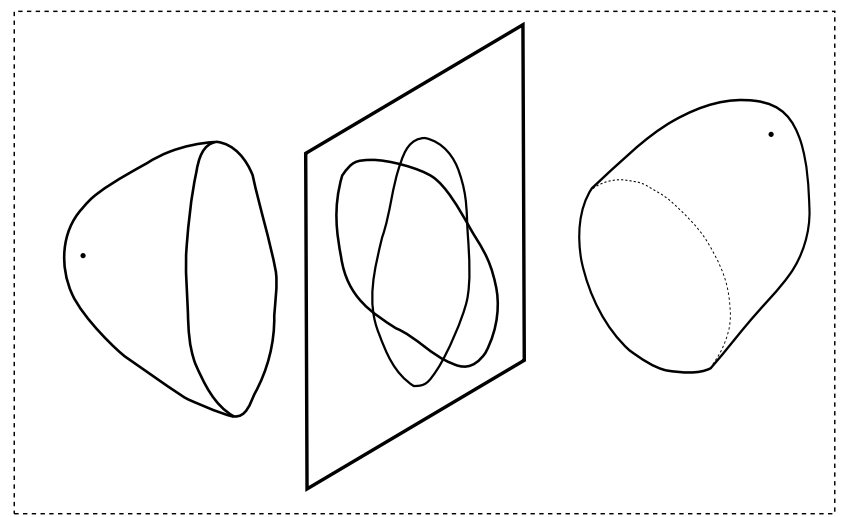

FIG. 6.1. Schematic of the "global first intersection" of the stable and unstable manifolds with the test section.

6. Computation of a Single Heteroclinic Intersection Point. In this section we develop some heuristic numerics for automatically generating the initial data $q, k_{u}$, and $k_{s}$ necessary to formulate Equation (4.2). We proceed in two steps; first a coarse heuristic computation which locates $k_{u}, k_{s}$ and an approximate intersection point $q_{0}$; then a Newton procedure which refines $q_{0}$ to an approximate intersection point $q$, valid to roughly machine precision.

6.1. Approximate Initial Data by Section. In order to determine how many iterates of the local stable and unstable manifolds are necessary in order to obtain an intersection, we exploit a particular cross section of the manifolds in phase space. Using this section we are able to read off the needed guesses.

Definition 6.1. Let $\ell$ be the line determined by $p_{0}$ and $p_{1}$. Let $p \in \ell$ be the point half way between $p_{0}$ and $p_{1}$. Let $T$ be the plane perpendicular to $\ell$ and containing $p$. We call $T$ the "half-way plane" for $p_{0}$ and $p_{1}$. Note that $T$ is unique.

Roughly speaking, we want to extend the local stable and unstable manifolds until they reach $T$ for the first time. A schematic situation is shown in Figure 6.1. We call the intersection of the two circles in Figure 6.1 the global first intersection of the stable and unstable manifolds relative to $T$, or simply the global first intersection. The following algorithm sketches the section computation.

Algorithm 2 (Compute Rough Section). Choose $D_{u}$ so that $P_{u}\left(D_{u}\right)=W_{l o c}^{u}\left(p_{0}\right)$ is entirely on the same side of $T$ as $p_{0}$. Fix $\varepsilon_{t o l}>0$.

- Discretize the parameter domains using $N$ points $\left\{x_{i}^{u}\right\}_{i=1}^{N} \subset D_{u}$. The points $W=\left\{P_{u}\left(x_{i}^{u}\right)\right\}$ discretize the local unstable manifold.

- Iterate the points $W$. Throw away iterates that cross T. Put the 'good'iterates in an array $S_{u}$.

- Let $I_{u}$ be the subcollection of $S_{u}$ within distance $\varepsilon_{\text {tol }}$ from the plane $T$.

Perform the same search for $W_{l o c}^{s}\left(p_{1}\right)$, yielding a set $I_{s}$. The sets $I_{u, s}$ from Algorithm 2 approximate (up to $\varepsilon_{t o l}$ ) the loops in the plane $T$ shown in Figure 6.1. The intersection 


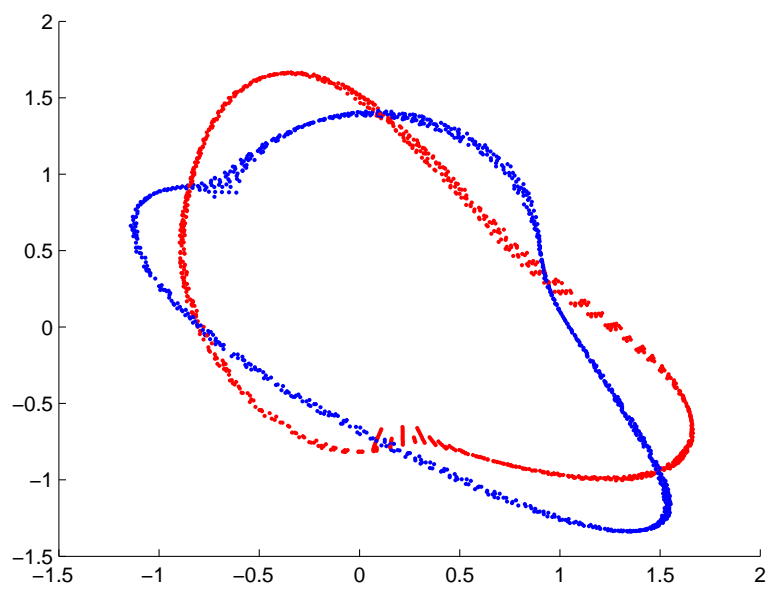

FIG. 6.2. Section of the Resonance Complex. The section suggests six intersection points.

of $I_{u, s}$ approximates the global first intersection. The following algorithm sketches the selection of the parameter guesses.

Algorithm 3 (Locate Parameter Guesses). Use the same $\varepsilon_{\text {tol }}$ as above, and choose a constant $C>1$.

- Let $Q$ be all pairs of points $\left(q^{u}, q^{s}\right) \in I_{u} \times I_{s}$ having $\left\|q^{u}-q^{s}\right\|<C \varepsilon_{\text {tol }}$.

- The points in $Q$ cluster into 'lumps' $Q_{i}$, where we say $q$ and $q$ ' are in the same 'lump' if $\left\|q-q^{\prime}\right\|<C \varepsilon_{\text {tol }}$.

- Choose representatives $\left(q_{i}^{u}, q_{i}^{s}\right) \in Q_{i}$.

- For each $i$ follow the point $q_{i}^{u}$ under iteration of $f^{-1}$ until the preimage is a point in $W=\left\{P_{u}\left(x_{i}^{u}\right)\right\}$. Let $k_{u_{i}}$ be the number of iterates necessary. Do the same for $q_{i}^{s}$ under $f$.

- Return the parameter guesses $x_{i}^{u}$ and $x_{i}^{s}$, as well as the iterate counts $k_{u_{i}}$ and $k_{s_{i}}$.

The 'lumps' approximate points in the intersection of $W^{u}\left(p_{0}\right)$ and $W^{s}\left(p_{1}\right)$. The parameter guesses have

$$
\left\|f^{k_{u_{i}}}\left(x_{i}^{u}\right)-f^{k_{s_{i}}}\left(x_{i}^{s}\right)\right\| \leq C \varepsilon_{t o l}
$$

In practice some experimentation with $C$ and $\varepsilon_{t o l}$, as well as with the density of the discretization points in $D_{s, u}$, is necessary in order to obtain a useful section. Note that since we are planning to input the resulting data into a Newton Method, the guesses need not be that good. An $\varepsilon_{t o l}$ on the order of $10^{-2}$ and $C=2$ are often sufficient to get the desired results.

EXAMPLE 6.1. Using the same parameter values, parameterizations, and radii of convergence as in Example 5.1, we compute a half-way section using the algorithms above. We choose a resolution $\varepsilon_{t o l}=2.5 \cdot 10^{-2}$.

This results, for example, in parameter values 


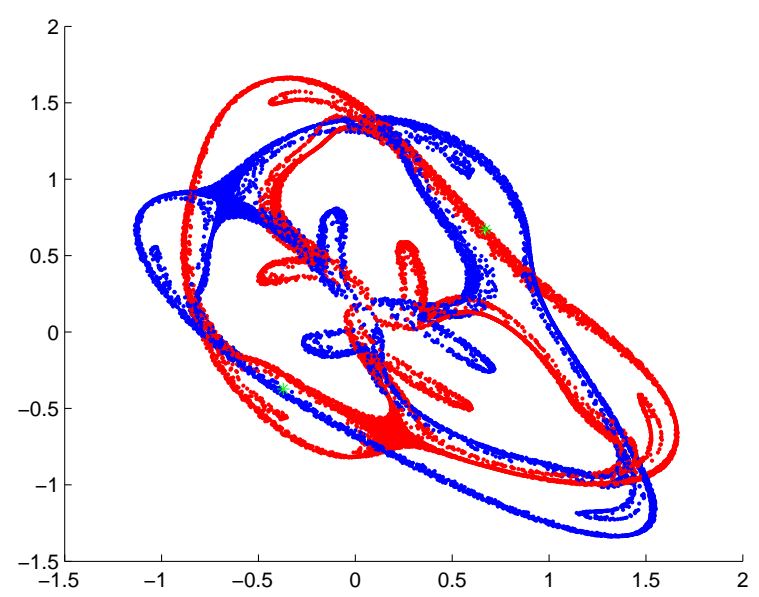

FIG. 6.3. Over-iterated Section of the Resonance Complex. Illustrates the accumulation of the two dimensional manifolds on the complementary one dimensional manifolds.

$$
x_{\text {guess }}^{u}=(-0.215,0.026) \in D_{u} \quad \text { and } \quad x_{\text {guess }}^{s}=(0.159,-0.014) \in D_{s}
$$

and iterate values $k_{u}=2, k_{s}=3$ so that

$$
||\left(f^{2} \circ P_{u}\right)\left(x_{\text {guess }}^{u}\right)-\left(f^{-3} \circ P_{s}\right)\left(x_{\text {guess }}^{s}\right)||<0.047
$$

The approximation to the global first intersection is shown in Figure 6.1. The Figure suggests that the first intersection has six arc components which pass through the half-way plane. Then, in addition to the approximate intersection given above, we can find five other distinct approximate intersections.

Our reason for focusing on the "first intersection" of the stable and unstable manifolds in phase space is illustrated in Figure (6.3). Here we see that unless we make a careful choice of section, we obtain too many intersection points to handle in an automated way. This is due to the $\lambda$-lemma.

6.2. Refining the Initial Guesses. The output of Algorithm 3 approximately solves the algebraic Equation (4.4). We apply a finite dimensional (degenerate) Newton Method, and obtain a solution accurate to almost machine precision (the linear operator in the Newton Method has one dimensional kernel, so that we invert as discussed in Section 2.1).

EXAMPLE 6.2. Beginning from the data $x_{\text {guess }}^{s}$ and $x_{\text {guess }}^{u}$ obtained in Example (6.1), the finite dimensional Newton Scheme

$$
\left(\begin{array}{l}
x_{n+1}^{u} \\
x_{n+1}^{s}
\end{array}\right)=\left(\begin{array}{c}
x_{n}^{u} \\
x_{n}^{s}
\end{array}\right)-\left[D F\left(x_{n}^{u}, x_{n}^{s}\right)\right]_{M P}^{-1} F\left(x_{n}^{u}, x_{n}^{s}\right)
$$


converges to

$$
x_{f}^{u}=(-0.21847150687026,0.02943166952031) \in D_{u}
$$

and

$$
x_{f}^{s}=(0.15932798404614,-0.00625895303931) \in D_{s}
$$

having

$$
\left\|\left(f^{2} \circ P_{u}\right)\left(x_{f}^{u}\right)-\left(f^{-3} \circ P_{s}\right)\left(x_{f}^{s}\right)\right\|<2.24 \times 10^{-16}
$$

Here $F\left(x^{u}, x^{s}\right)$ is as in Section 6, and the degeneracy of the linear operator in the the Newton scheme requires the computation of a Moore Penrose Inverse as discussed in Section 2.1.

If we then let $q=f^{2}\left(P_{u}\left(x_{f}^{u}\right)\right)$, then we have

$$
q=\left(\begin{array}{c}
0.105778203679722 \\
1.398309723465859 \\
-1.050157418426429
\end{array}\right)
$$

as an approximate heteroclinic point in phase space. One can check the validity of the approximation by iterating $q$. We note for example that

$$
\left\|p_{1}-f^{77}(q)\right\|<8.8 \times 10^{-6},
$$

so that $q$ has the correct asymptotic behavior for more than 75 iterates (and similarly for inverse iterates). Figure 7-ab illustrate the orbits of the results in both parameter space and phase space.

7. Numerical Results of the Arc Computation. In this section we give numerical results for step 3 of Algorithm 1. These depend on the results from Examples 5.1, 6.1, and 6.2. We run the Newton scheme developed in Section 4 with

$$
\begin{gathered}
\gamma_{0}(\theta)=\left(\begin{array}{c}
x^{u} \\
x^{s}
\end{array}\right)+\theta v= \\
\left(\begin{array}{c}
-0.21847150687026 \\
0.02943166952031 \\
0.15932798404614 \\
-0.00625895303931
\end{array}\right)+\theta\left(\begin{array}{c}
-0.11086390724073 \\
-0.42395453452640 \\
-0.24077289633209 \\
-3.988955692034501 \times 10^{-4}
\end{array}\right)
\end{gathered}
$$

where $v$ is chosen so that $v \in \operatorname{ker}(D F)$ as discussed in Section 4.1. With truncated formal series of order one hundred, the Newton scheme numerically converges after seven iterates to a curve $\gamma=\left(\gamma_{u}, \gamma_{s}\right)$, for which we check that

$$
\sup _{\theta \in[-0.5,0.5]}\left\|\left[f^{2} \circ P_{u} \circ \gamma_{u}\right](\theta)-\left[f^{-3} \circ P_{s} \circ \gamma_{s}\right](\theta)\right\|<5 \times 10^{-14}
$$


Then $\gamma$ has $\varepsilon$-numerical radius of convergence of $r=0.5$, with $\varepsilon$ equal to roughly 200 multiples of double precision machine epsilon. Figure 7.1-c. shows the arc $\gamma^{u}$ through $x_{0}^{u}$ in parameter space. The image of $\gamma^{s}$ in stable parameter space is similar. The image $f^{2} \circ P_{u} \circ \gamma^{u}=\tilde{\gamma}$ in phase space is shown in Figure 7.1-d.

REMARKS 7.1. Once we have obtained an accurate computation of the local arc segment $\gamma$, this local data can be iterated to obtain information about the global dynamics. This however gives rise to an interesting complication.

a) Given an arc $s$, we say that the arc $\hat{s}$ continues $s$ if $s \cap \hat{s} \neq \emptyset$. Figure 7 illustrates the fact that there is no invariant continuation of $\gamma^{u}$. To see this note in (d) that $E_{\lambda}^{-3} \gamma^{u}$ (green arc) does in fact continue $\gamma^{u}$ (black arc). Nevertheless, $E_{\lambda}^{-1} \gamma^{u}$, and $E_{\lambda}^{-2} \gamma^{u}$ do not lie on any continuation of $\gamma^{u}$; hence the continuation of $\gamma^{u}$ is not invariant.

b) Let

$$
S=\overline{\bigcup_{k=-\infty}^{\infty} f^{k}(\tilde{\gamma})}
$$

$S$ is approximated by green set in Figure 7.3. $S$ is globally invariant, and if $p \in S$ then $p$ is either heteroclinic from $p_{0}$ to $p_{1}$, or $p$ is fixed. We call $S$ a branched heteroclinic manifold.

c) Note that the branched heteroclinic manifold in Figure 7.3 passes through three of the six "first intersection points" found in Figure 6.1. By repeating the analysis illustrated in the Examples 6.1, and 6.2 for one of the three remaining first intersection points shown in Figure 7.4, we obtain the complete branched heteroclinic manifold of first intersection shown in Figure 7.4.

d) Note that, as in [30, 28], the stable and unstable manifolds of $p_{0}$ and $p_{1}$ enclose a compact neighborhood $R \subset \mathbb{R}^{3}$. Such a region $R$ is sometimes called a resonance zone. The heteroclinic branched manifold illustrated in Figure 7.4 is needed in order to define certain geometric invariants introduced in [31]. These geometric invariants are related to the dynamics in $R$ and extend the planar techniques developed in [28] to volume preserving diffeomorphisms. Numerical computation of these geometric invariants will be aided by exploiting the techniques developed here. More precisely, the geometric invariants of [31] are defined in terms of certain line integrals over the branched heteroclinic manifold of first intersection. Since we have computed the intersection to high precision we hope to make an accurate numerical study of these line integrals, in order to better understand the relation between the integrals and the dynamics of the map.

8. Acknowledgements. The authors would like to thank J.T. Halbert for carefully reading an earlier draft of the manuscript and many helpful comments and corrections. JDMJ was supported by NSF grant DMS 0354567 and by the University of Texas Department of Mathematics Program In Applied and Computational Analysis RTG Fellowship during this work. This paper was written while HL held a Research Visiting Scholar position at the University of Texas at Austin and was supported in part by Asociación Mexicana de Cultura and CONACYT-Mexico. Useful conversa- 
a
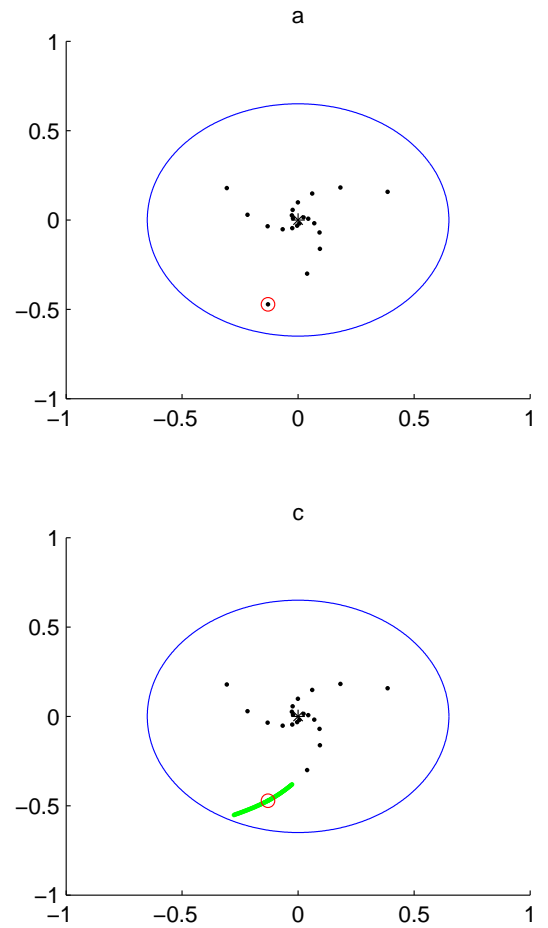

b

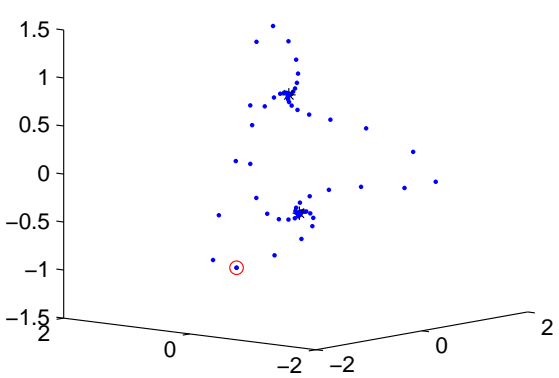

$d$

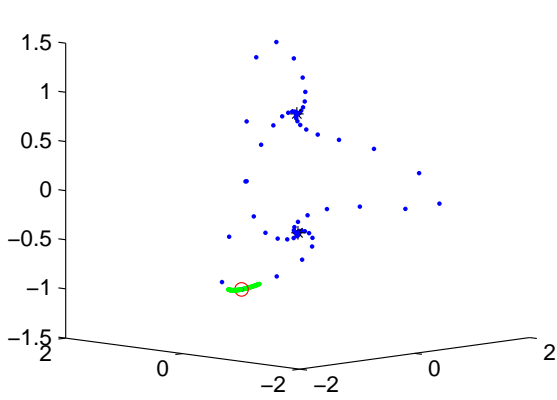

FIG. 7.1. Heteroclinic Orbits: (a) shows the unstable parameter domain $D_{u}$ with a blue circle. The red circle highlights an unstable parameter value $x_{u}$ for a heteroclinic point $q$. The black dots are the backward orbit of $x_{u}$ in parameter space under the linear dynamics $E_{\lambda_{u}}^{-1}$ (in backward time the point converges to the origin) (b) shows the lift $q=P_{u}\left(x_{u}\right)$ in phase space. Now $q$ is highlighted with a red circle. The blue dots show 50 forward and backward iterates of the highlighted point under the volume preserving Hénon dynamics. The highlighted point converges to the fixed point $p_{0}$ in backward, and $p_{1}$ in forward time. (c) The green curve is an heteroclinic parameter arc $\gamma^{u}$ through the highlighted point $x_{u}$. This arc is computed using the Newton method of Section 4. (d) Shows the image of the parameter arc $\gamma^{u}$ under the parameterization map $P_{u}$. This results in a heteroclinic arc $\tilde{\gamma} \subset W^{u}\left(p_{0}\right) \cap W^{s}\left(p_{1}\right)$ in phase space

tions with Jim Meiss and Rafael de la Llave are gratefully acknowledged.

\section{Appendix A. Power series.}

A.1. Product formula and the convolution of coefficients. A general power series of two variables can be written as

$$
P(x, y)=a_{00}+a_{10} x+a_{01} y+a_{20} x^{2}+a_{11} x y+a_{02} y^{2}+\ldots=\sum_{j=0}^{\infty} \sum_{i=0}^{\infty} a_{i j} x^{i} y^{j}
$$

where the subscript ij of $a_{i j}$ counts the number of powers of $x$ and $y$ in each term.

If $P$ and $Q$ are power series of two variables then we define formally $P+Q$ and $c \cdot P$, with $c$ a scalar, in the obvious way. Let 

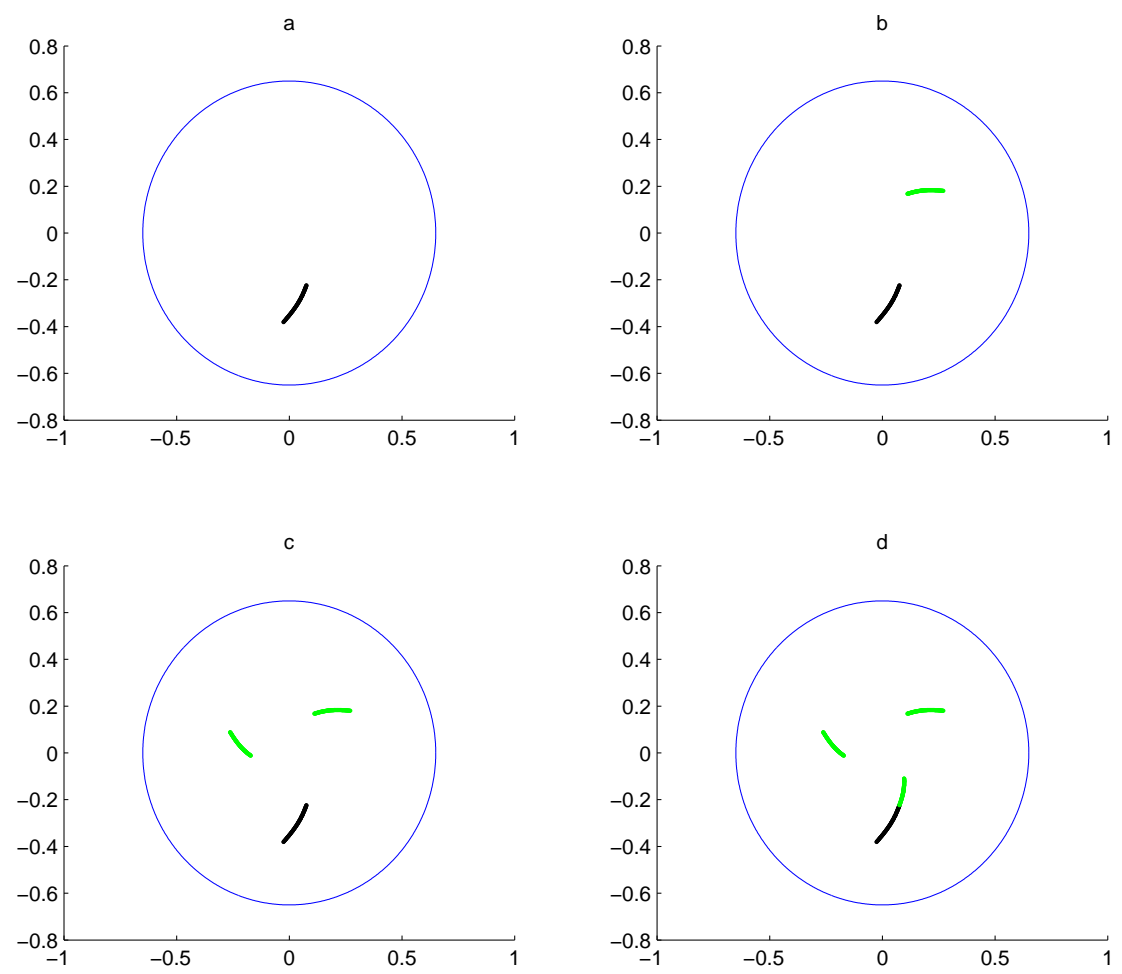

FIG. 7.2. Dynamics of Heteroclinic Arcs: (a) shows the unstable parameter arc $\gamma^{u} \subset D_{u}$ in black and the boundary of the parameter disk $D_{u}$ in blue. (b) shows the arc $\gamma^{u}$ in black as well as its first inverse iterate $E_{\lambda_{u}}^{-1}\left(\gamma^{u}\right)$ in green. (c) shows $\gamma^{u}$ in black, as well as $E_{\lambda_{u}}^{-1}\left(\gamma^{u}\right)$ and $E_{\lambda_{u}}^{-2}\left(\gamma^{u}\right)$ in green. (d) shows $\gamma^{u}$ in black as well as its first three inverse iterates in green. Note that $\gamma^{u} \cup E_{\lambda_{u}}^{-3}\left(\gamma^{u}\right)$ is a continuation of the curve $\gamma^{u}$. Observe that if we continue a heteroclinic arc $\gamma^{u}$ all the way to the fixed points, the resulting curve is not invariant. To obtain an invariant set it is necessary to include the continuations of the first and second iterates of $\gamma^{u}$ as well (as illustrated in the next figure).

$$
P(x, y)=\sum_{j=0}^{\infty} \sum_{i=0}^{\infty} a_{i j} x^{i} y^{j} \quad Q(x, y)=\sum_{j=0}^{\infty} \sum_{i=0}^{\infty} b_{i j} x^{i} y^{j}
$$

and define

$$
(P+Q)(x, y)=\sum_{j=0}^{\infty} \sum_{i=0}^{\infty}\left(a_{i j}+b_{i j}\right) x^{i} y^{j}
$$

and

$$
(c \cdot P)(x, y)=\sum_{j=0}^{\infty} \sum_{i=0}^{\infty} c a_{i j} x^{i} y^{j}
$$




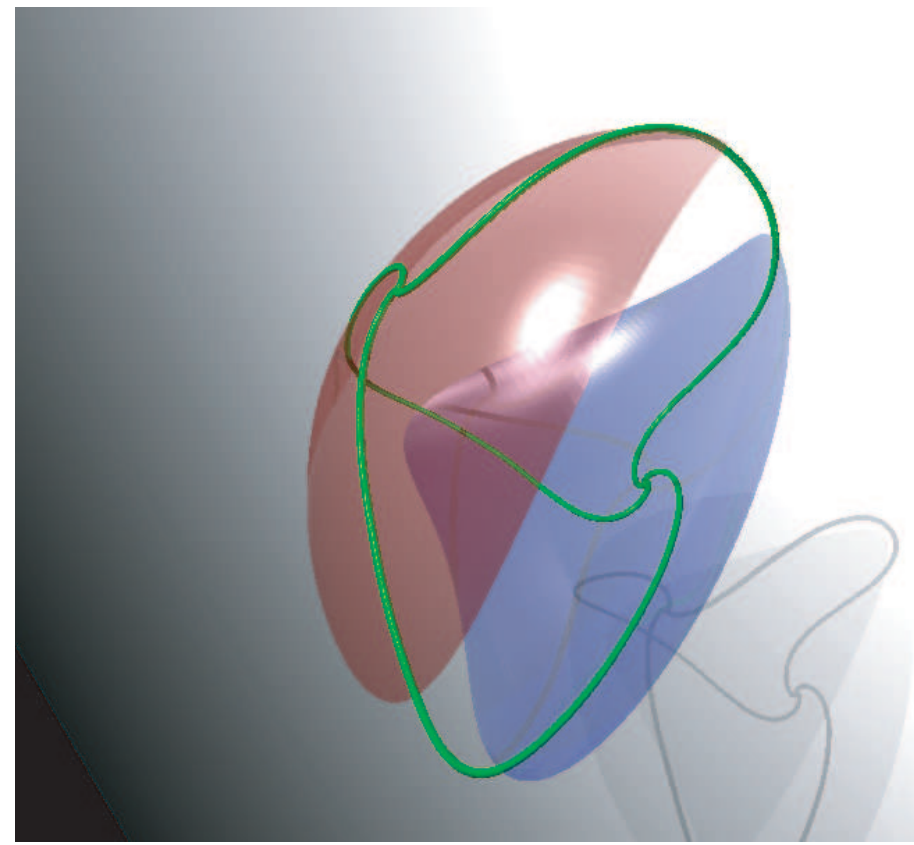

Fig. 7.3. Heteroclinic Branched Manifold: $W_{l o c}^{u}\left(p_{0}\right)$ in blue and $W_{l o c}^{s}\left(p_{1}\right)$ in red. The green curves approximate the invariant set $S$ defined in Equation (7.1) and are computed by $\bigcup_{k=-50}^{50} f^{k}(\tilde{\gamma})$. The Figure also illustrates the accuracy of the computation of the initial arc $\gamma^{u}$. The initial arc segment shows the correct asymptotic behavior even after being iterated many times.

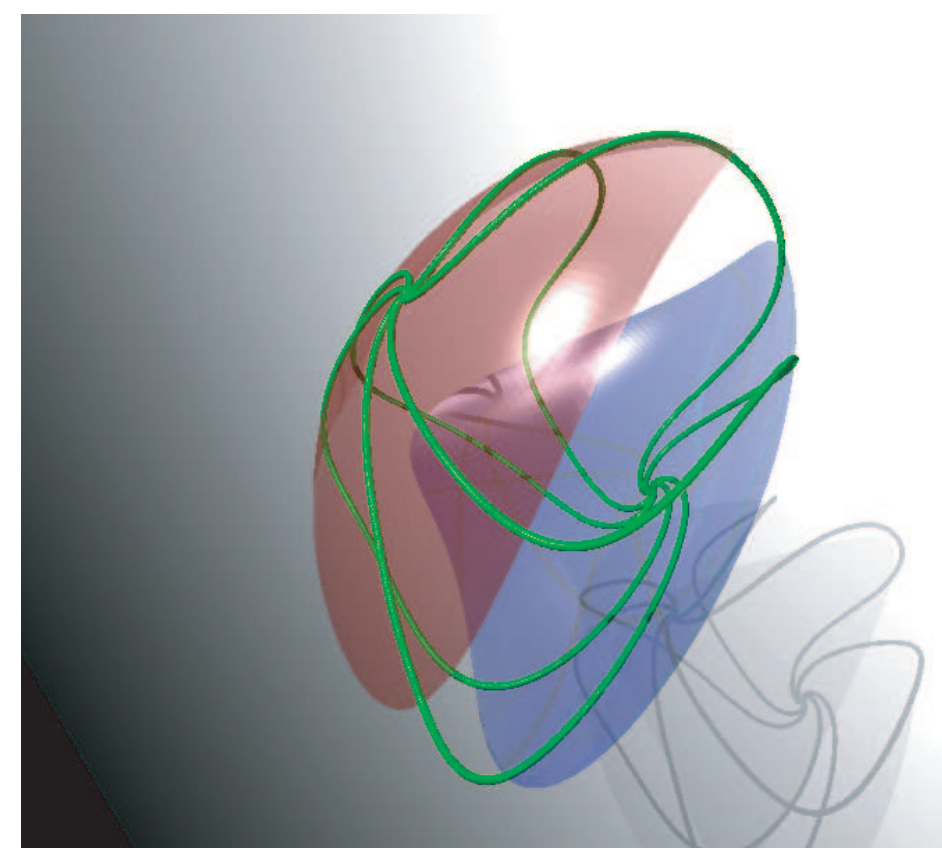

FIG. 7.4. Complete "first intersection" of $W^{u}\left(p_{0}\right)$ and $W^{s}\left(p_{1}\right)$ as discussed in Remark c. 
The Cauchy product of the series is defined to be

$$
\begin{gathered}
(Q \cdot P)(x, y)=\sum_{j=0}^{\infty} \gamma_{j}(x) y^{j} \\
=\sum_{j=0}^{\infty} \sum_{k=0}^{j} \alpha_{j-k}(x) \beta_{k}(x) y^{j}=\sum_{j=0}^{\infty} \sum_{k=0}^{j} \sum_{i=0}^{\infty} c_{i(j-k)} x^{i} y^{j} \\
=\sum_{j=0}^{\infty} \sum_{k=0}^{j} \sum_{i=0}^{\infty} \sum_{l=0}^{i} a_{(i-l)(j-k)} b_{l k} x^{i} y^{j} \\
=\sum_{j=0}^{\infty} \sum_{i=0}^{\infty} \sum_{k=0}^{j} \sum_{l=0}^{i} a_{(i-l)(j-k)} b_{l k} x^{i} y^{j}
\end{gathered}
$$

A.2. A Composition Formula. Let $G: \mathbb{R}^{2} \rightarrow \mathbb{R}$, and $\gamma: \mathbb{R} \rightarrow \mathbb{R}^{2}$ be given by

$$
G(s, t)=\sum_{n=0}^{\infty} \sum_{m=0}^{\infty}(s+i t)^{m}(s-i t)^{n} a_{m n}
$$

and

$$
\gamma(\theta)=\left(\begin{array}{c}
s(\theta) \\
t(\theta)
\end{array}\right)=\sum_{j=0}^{\infty} \theta^{j}\left(\begin{array}{c}
\alpha_{j} \\
\beta_{j}
\end{array}\right) .
$$

Then the composition $G \circ \gamma: \mathbb{R} \rightarrow \mathbb{R}$ has formal series

$$
\sum_{j=0}^{\infty} A_{j} \theta^{j}=G \circ \gamma(\theta) .
$$

We want a recursive formula for the coefficients $A_{j}$. We compute

$$
\begin{gathered}
G \circ \gamma(t)=\sum_{n=0}^{\infty} \sum_{m=0}^{\infty}[s(\theta)+i t(\theta)]^{m}[s(\theta)-i t(\theta)]^{n} a_{m n} \\
=\sum_{n=0}^{\infty} \sum_{m=0}^{\infty}\left[\sum_{j=0}^{\infty} \alpha_{j} \theta^{j}+i \sum_{j=0}^{\infty} \beta_{j} \theta^{j}\right]^{m}\left[\sum_{j=0}^{\infty} \alpha_{j} \theta^{j}-i \sum_{j=0}^{\infty} \beta_{j} \theta^{j}\right]^{n} a_{m n} \\
=\sum_{n=0}^{\infty} \sum_{m=0}^{\infty}\left[\sum_{j=0}^{\infty}\left(\alpha_{j}+i \beta_{j}\right) \theta^{j}\right]^{m}\left[\sum_{j=0}^{\infty}\left(\alpha_{j}-i \beta_{j}\right) \theta^{j}\right]^{n} a_{m n} .
\end{gathered}
$$


Denote

$$
\sum_{j=0}^{\infty}\left[a_{j}(m)+i b_{j}(m)\right] \theta^{j}=\left[\sum_{j=0}^{\infty}\left(\alpha_{j}+i \beta_{j}\right) \theta^{j}\right]^{m}
$$

and

$$
\sum_{j=0}^{\infty}\left[c_{j}(n)+i d_{j}(n)\right] \theta^{j}=\left[\sum_{j=0}^{\infty}\left(\alpha_{j}-i \beta_{j}\right) \theta^{j}\right]^{n} .
$$

Then

$$
\begin{aligned}
G \circ \gamma(\theta) & =\sum_{n=0}^{\infty} \sum_{m=0}^{\infty} a_{m n}\left(\sum_{j=0}^{\infty}\left[a_{j}(m)+i b_{j}(m)\right]\right)\left(\sum_{j=0}^{\infty}\left[c_{j}(n)+i d_{j}(n)\right]\right) \\
& =\sum_{n=0}^{\infty} \sum_{m=0}^{\infty} a_{m n} \sum_{j=0}^{\infty} \sum_{k=0}^{j}\left(a_{k}(m) c_{j-k}(n)-b_{k}(m) d_{j-k}(n)\right) \theta^{j} \\
& +i \sum_{n=0}^{\infty} \sum_{m=0}^{\infty} a_{m n} \sum_{j=0}^{\infty} \sum_{k=0}^{j}\left(a_{k}(m) d_{j-k}(n)+b_{k}(m) c_{j-k}(n)\right) \theta^{j} .
\end{aligned}
$$

Let $a_{m n}=x_{m n}+i y_{m n}$. Then the real part is

$$
\begin{gathered}
G \circ \gamma(\theta)=\sum_{n=0}^{\infty} \sum_{m=0}^{\infty} x_{m n} \sum_{j=0}^{\infty} \sum_{k=0}^{j}\left(a_{k}(m) c_{j-k}(n)-b_{k}(m) d_{j-k}(n)\right) \theta^{j} \\
-\sum_{n=0}^{\infty} \sum_{m=0}^{\infty} y_{m n} \sum_{j=0}^{\infty} \sum_{k=0}^{j}\left(a_{k}(m) d_{j-k}(n)+b_{k}(m) c_{j-k}(n)\right) \theta^{j} \\
=\sum_{j=0}^{\infty}\left(\sum_{n=0}^{\infty} \sum_{m=0}^{\infty} \sum_{k=0}^{j} \Xi_{k, j}(m, n)\right) \theta^{j}
\end{gathered}
$$

where

$$
\begin{gathered}
\Xi_{k, j}(m, n)= \\
x_{m n}\left[a_{k}(m) c_{j-k}(n)-b_{k}(m) d_{j-k}(n)\right]-y_{m n}\left[a_{k}(m) d_{j-k}(n)+b_{k}(m) c_{j-k}(n)\right] .
\end{gathered}
$$

Recall here that $a_{j}(n), b_{j}(n), c_{j}(n)$, and $d_{j}(n)$ are series coefficients for the real and imaginary parts of the series expansions of $\gamma^{n}$ and $\bar{\gamma}^{n}$. Rather than give recurrence relations for these coefficients, we give an algorithm which computes them.

Algorithm 4 (Compute Coefficients of Powers Through N). 
function computePowersCoefficients $\left(\left\{\alpha_{j}\right\},\left\{\beta_{j}\right\}, N\right)$;

$$
\begin{aligned}
& \left\{a_{j}(1)\right\}=\left\{\alpha_{j}\right\} ; \\
& \left\{b_{j}(1)\right\}=\left\{\beta_{j}\right\} ; \\
& \left\{a_{j}(2)\right\}=\left\{\alpha_{j}\right\} *\left\{\alpha_{j}\right\}-\left\{\beta_{j}\right\} *\left\{\beta_{j}\right\} ; \\
& \left\{b_{j}(2)\right\}=\left\{\alpha_{j}\right\} *\left\{\beta_{j}\right\}+\left\{\beta_{j}\right\} *\left\{\alpha_{j}\right\} ; \\
& \text { for }(3 \leq k \leq N) \\
& \quad p=\operatorname{ceil}(k / 2) ; \\
& \quad q=\operatorname{integerPart}(k / 2) ; \\
& \quad\left\{a_{j}(k)\right\}=\left\{a_{j}(p)\right\} *\left\{a_{j}(q)\right\}-\left\{b_{j}(p)\right\} *\left\{b_{j}(q)\right\} ; \\
& \quad\left\{b_{j}(k)\right\}=\left\{a_{j}(p)\right\} *\left\{b_{j}(q)\right\}+\left\{b_{j}(p)\right\} *\left\{a_{j}(q)\right\} ; \\
& \text { end for }
\end{aligned}
$$

return $\left(\left\{a_{j}(n)\right\},\left\{b_{j}(n)\right\}\right)$;

The input to the algorithm is the list of the power series coefficients for $\gamma$. The output is a matrix whose $k$-th column is a list of the power series coefficients of $\gamma^{n}$.* denotes series coefficient convolution. The algorithm computes all powers of $\gamma$ through $N$ with a minimum number of convolutions. Calling the algorithm with input $\alpha_{j}$ and $-\beta_{j}$ gives the coefficients for $\bar{\gamma}$. Using this algorithm we compute the coefficients of the powers of $\gamma$ and its conjugate before beginning the composition computation. The recursion relations for $\Xi$ allow direct computation of the composition coefficients once the power coefficients are know.

\section{REFERENCES}

[1] K. Alligood, E. Sander, and J. Yorke. Three dimensional crisis: Crossing bifurcations and unstable dimension variability. Phys. Rev. Lett., 96, 2006.

[2] K. T. Alligood, E. Sander, and J. A. Yorke. Explosions in dimensions one through three. Rend. Semin. Mat. Univ. Politec. Torino, 65(1):1-15, 2007.

[3] I. Baldomá and T. M. Seara. Breakdown of heteroclinic orbits for some analytic unfoldings of the Hopf-zero singularity. J. Nonlinear Sci., 16(6):543-582, 2006.

[4] J. M. Bergamin, T. Bountis, and M. N. Vrahatis. Homoclinic orbits of invertible maps. Nonlinearity, 15(5):1603-1619, 2002.

[5] M. Berz and K. Makino. Cosy infinity. http://www.cosyinfinity.org.

[6] M. Berz and K. Makino. Suppression of the wrapping effect by taylor model- based validated integrators. MSU HEP Report 40910 (2003).

[7] Martin Berz and Georg Hoffstätter. Computation and application of Taylor polynomials with interval remainder bounds. Reliab. Comput., 4(1):83-97, 1998.

[8] W.-J. Beyn and J.-M. Kleinkauf. The numerical computation of homoclinic orbits for maps. SIAM J. Numer. Anal., 34(3):1207-1236, 1997.

[9] Henk Broer. Formal normal form theorems for vector fields and some consequences for bifurcations in the volume preserving case. In Dynamical systems and turbulence, Warwick 1980 (Coventry, 1979/1980), volume 898 of Lecture Notes in Math., pages 54-74. Springer, Berlin, 1981.

[10] H. Martin Bücker and George F. Corliss. A bibliography of automatic differentiation. In Automatic differentiation: applications, theory, and implementations, volume 50 of Lect. Notes Comput. Sci. Eng., pages 321-322. Springer, Berlin, 2006.

[11] Xavier Cabré, Ernest Fontich, and Rafael de la Llave. The parameterization method for invariant manifolds. I. Manifolds associated to non-resonant subspaces. Indiana Univ. Math. J., 52(2):283-328, 2003.

[12] Xavier Cabré, Ernest Fontich, and Rafael de la Llave. The parameterization method for invariant manifolds. III. Overview and applications. J. Differential Equations, 218(2):444-515, 
2005.

[13] Brian A. Coomes, Hüseyin Koçak, and Kenneth J. Palmer. Homoclinic shadowing. J. Dynam. Differential Equations, 17(1):175-215, 2005.

[14] Lorenzo J. Díaz and Jorge Rocha. Nonconnected heterodimensional cycles: bifurcation and stability. Nonlinearity, 5(6):1315-1341, 1992.

[15] E. J. Doedel, B. W. Kooi, G. A. K. van Voorn, and Yu. A. Kuznetsov. Continuation of connecting orbits in 3D-ODEs. I. Point-to-cycle connections. Internat. J. Bifur. Chaos Appl. Sci. Engrg., 18(7):1889-1903, 2008.

[16] Eusebius J. Doedel and Mark J. Friedman. Numerical computation of heteroclinic orbits. J. Comput. Appl. Math., 26(1-2):155-170, 1989. Continuation techniques and bifurcation problems.

[17] H. R. Dullin and J. D. Meiss. Quadratic volume-preserving maps: invariant circles and bifurcations. SIAM J. Appl. Dyn. Syst., 8(1):76-128, 2009.

[18] J. P. England, B. Krauskopf, and H. M. Osinga. Computing one-dimensional global manifolds of Poincaré maps by continuation. SIAM J. Appl. Dyn. Syst., 4(4):1008-1041 (electronic), 2005.

[19] Victor Guillemin and Alan Pollack. Differential topology. Prentice-Hall Inc., Englewood Cliffs, N.J., 1974.

[20] Philip J. Holmes and Jerrold E. Marsden. Melnikov's method and Arnol'd diffusion for perturbations of integrable Hamiltonian systems. J. Math. Phys., 23(4):669-675, 1982.

[21] Thorsten Hüls. Bifurcation of connecting orbits with one nonhyperbolic fixed point for maps. SIAM J. Appl. Dyn. Syst., 4(4):985-1007 (electronic), 2005.

[22] Àngel Jorba and Mercè Ollé. Invariant curves near Hamiltonian-Hopf bifurcations of fourdimensional symplectic maps. Nonlinearity, 17(2):691-710, 2004.

[23] Donald E. Knuth. The art of computer programming. Vol. 2. Addison-Wesley Publishing Co., Reading, Mass., third edition, 1997. Seminumerical algorithms, Addison-Wesley Series in Computer Science and Information Processing.

[24] B Krauskopf and H Osinga. Globalizing two-dimensional unstable manifolds of maps. Int. Journal of Bifurcation and Chaos, 8(3):483-503, 1998.

[25] B. Krauskopf, H. M. Osinga, E. J. Doedel, M. E. Henderson, J. Guckenheimer, A. Vladimirsky, M. Dellnitz, and O. Junge. A survey of methods for computing (un)stable manifolds of vector fields. Internat. J. Bifur. Chaos Appl. Sci. Engrg., 15(3):763-791, 2005.

[26] Bernd Krauskopf and Thorsten Rieß. A Lin's method approach to finding and continuing heteroclinic connections involving periodic orbits. Nonlinearity, 21(8):1655-1690, 2008.

[27] Héctor E. Lomelí and James D. Meiss. Quadratic volume-preserving maps. Nonlinearity, 11(3):557-574, 1998.

[28] Héctor E. Lomelí and James D. Meiss. Heteroclinic orbits and flux in a perturbed integrable Suris map. Phys. Lett. A, 269(5-6):309-318, 2000.

[29] Héctor E. Lomelí and James D. Meiss. Heteroclinic primary intersections and codimension one Melnikov method for volume-preserving maps. Chaos, 10(1):109-121, 2000. Chaotic kinetics and transport (New York, 1998).

[30] Héctor E. Lomelí and James D. Meiss. Heteroclinic primary intersections and codimension one Melnikov method for volume-preserving maps. Chaos, 10(1):109-121, 2000. Chaotic kinetics and transport (New York, 1998).

[31] Héctor E. Lomelí and James D. Meiss. Resonance zones and lobe volumes for exact volumepreserving maps. Nonlinearity, 22(8):1761-1789, 2009.

[32] Kyoko Makino and Martin Berz. Taylor models and other validated functional inclusion methods. Int. J. Pure Appl. Math., 6(3):239-316, 2003.

[33] V. K. Mel'nikov. On the stability of a center for time-periodic perturbations. Trudy Moskov. Mat. Obšč., 12:3-52, 1963. Trans. Moscow Math. Soc.

[34] J. Milnor. Morse theory. Based on lecture notes by M. Spivak and R. Wells. Annals of Mathematics Studies, No. 51. Princeton University Press, Princeton, N.J., 1963.

[35] S. Newhouse, M. Berz, J. Grote, and K. Makino. On the estimation of topological entropy on surfaces. In Geometric and probabilistic structures in dynamics, volume 469 of Contemp. Math., pages 243-270. Amer. Math. Soc., Providence, RI, 2008.

[36] J. M. Ortega and W. C. Rheinboldt. Iterative solution of nonlinear equations in several variables, volume 30 of Classics in Applied Mathematics. Society for Industrial and Applied 
Mathematics (SIAM), Philadelphia, PA, 2000. Reprint of the 1970 original.

[37] Jacob Palis, Jr. and Welington de Melo. Geometric theory of dynamical systems. SpringerVerlag, New York, 1982. An introduction, Translated from the Portuguese by A. K. Manning.

[38] H. Poincaré. Les méthodes nouvelles de la mécanique céleste. Tome I-III. Les Grands Classiques Gauthier-Villars. Librairie Scientifique et Technique Albert Blanchard, Paris, 1987. Reprint of the 1899 original.

[39] Christian Potzsche and Martin Rasmussen. Taylor approximation of integral manifolds. Journal of Dynamics and Differential Equations, 18(2):427-460, 2006.

[40] W. C. Rheinboldt. MANPAK: a set of algorithms for computations on implicitly defined manifolds. Comput. Math. Appl., 32(12):15-28, 1996.

[41] Werner C. Rheinboldt. Numerical analysis of parametrized nonlinear equations. University of Arkansas Lecture Notes in the Mathematical Sciences, 7. John Wiley \& Sons Inc., New York, 1986. A Wiley-Interscience Publication.

[42] Werner C. Rheinboldt. On the computation of multidimensional solution manifolds of parametrized equations. Numer. Math., 53(1-2):165-181, 1988.

[43] Dietmar Salamon. Morse theory, the Conley index and Floer homology. Bull. London Math. Soc., 22(2):113-140, 1990.

[44] Matthias Schwarz. Morse homology, volume 111 of Progress in Mathematics. Birkhäuser Verlag, Basel, 1993.

[45] Gilbert Strang. Linear algebra and its applications. Thomson (Brooks/Cole), New York, third edition, 1988.

[46] Kazuyuki Yagasaki, Alan R. Champneys, and Boris A. Malomed. Discrete embedded solitons. Nonlinearity, 18(6):2591-2613, 2005.

[47] Yongkui Zou and Wolf-Jürgen Beyn. On the existence of transversal heteroclinic orbits in discretized dynamical systems. Nonlinearity, 17(6):2275-2292, 2004. 